\title{
Inborn Errors of Energy Metabolism Associated with Myopathies
}

\author{
Anibh M. Das, ${ }^{1}$ Ulrike Steuerwald, ${ }^{2}$ and Sabine Illsinger ${ }^{1}$ \\ ${ }^{1}$ Department of Paediatric Kidney-, Liver- and Metabolic Diseases, Hannover Medical School, Carl Neuberg Street 1, \\ 30625 Hannover, Germany \\ ${ }^{2}$ Screening Laboratory Hannover, Steinweg 13b, 30952 Ronnenberg, Germany \\ Correspondence should be addressed to Anibh M. Das, das.anibh@mh-hannover.de
}

Received 29 October 2009; Revised 19 January 2010; Accepted 22 February 2010

Academic Editor: Henk L. M. Granzier

Copyright () 2010 Anibh M. Das et al. This is an open access article distributed under the Creative Commons Attribution License, which permits unrestricted use, distribution, and reproduction in any medium, provided the original work is properly cited.

Inherited neuromuscular disorders affect approximately one in 3,500 children. Structural muscular defects are most common; however functional impairment of skeletal and cardiac muscle in both children and adults may be caused by inborn errors of energy metabolism as well. Patients suffering from metabolic myopathies due to compromised energy metabolism may present with exercise intolerance, muscle pain, reversible or progressive muscle weakness, and myoglobinuria. In this review, the physiology of energy metabolism in muscle is described, followed by the presentation of distinct disorders affecting skeletal and cardiac muscle: glycogen storage diseases types III, V, VII, fatty acid oxidation defects, and respiratory chain defects (i.e., mitochondriopathies). The diagnostic work-up and therapeutic options in these disorders are discussed.

\section{Introduction}

Both skeletal and heart muscle are highly dependent on energy supply. As energy demand of these muscle tissues varies by several orders of magnitude, energy metabolism has to be tightly regulated in order to meet varying energy requirement.

In this review, we shall first describe the physiology of energy metabolism in muscle, and then deal with different myopathies caused by inborn errors of energy metabolism.

\section{Physiology of Energy Metabolism in Muscle}

Glucose, fatty acids, and, to a lesser extent, amino acids serve as energy substrates for skeletal muscle cells. During quiescence, fatty acids are the main fuel. However, glucose and 6 amino acids (leucine, isoleucine, valine, asparagine, aspartate, glutamate) may also be used by the resting muscle $[1,2]$. Only leucine and isoleucine can be oxidised in muscle after being converted to acetyl CoA. The other amino acids are used solely for de novo synthesis of citric acid cycleintermediates.

Glycogen is stored in skeletal and heart muscle. In contrast to liver glycogen, muscle glycogen does not serve glucose homeostasis in the body but is almost exclusively used for energy metabolism in muscle itself. In the initial phase of exercise, blood supply to skeletal muscle is not yet adequate; hence oxygen and blood-born energy substrates (i.e., fatty acids) are lacking. In this early phase, muscle relies on its own energy reserves, that is, muscle glycogen, and generates energy via anaerobic pathways. After a few minutes, blood vessels dilate, blood flow rises, and muscle cells are provided with oxygen and blood-born substrates. During this phase of exercise, muscle relies on aerobic energy generation via oxidative phosphorylation, mainly from fatty acids, but also (to a lesser extent) glucose and amino acids.

Fatty acids are metabolized in the mitochondrial matrix to acetyl-CoA via beta-oxidation (Figures 1 and 2). Fatty acids are the major source of energy in man, especially during fasting. Most tissues are able to oxidize fatty acids to $\mathrm{CO}_{2}$ and water. In addition, liver has the capacity to synthesize ketone bodies (acetoacetate and 3-hydroxybutyrate from acetyl-CoA), which serve as an important fuel for extrahepatic organs, especially the brain, during catabolism. A rate-limiting step in mitochondrial fatty acid oxidation is the carnitine-palmitoyltransferase 1 (CPT1) reaction [3]. Adequate glucose supply, conversion of acetylCoA to malonyl-CoA by acetyl-CoA carboxylase, and the concomitant inhibition of CPT1 reduce long-chain fatty 


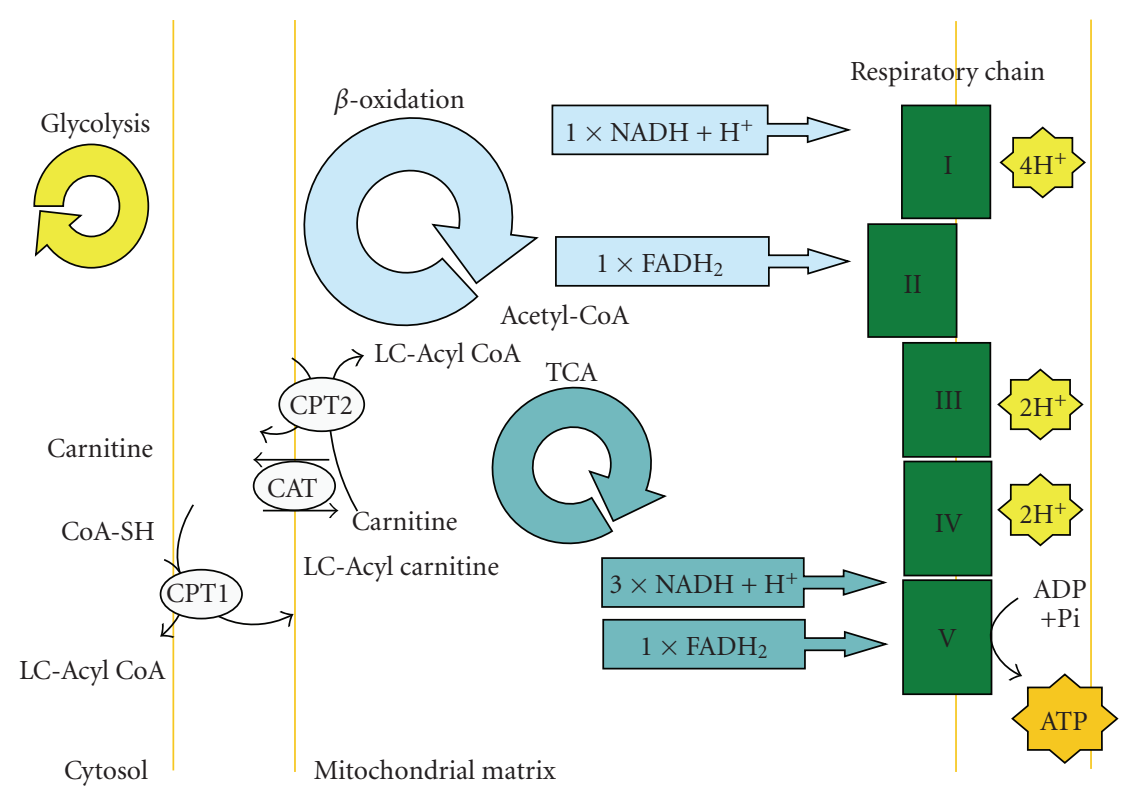

FIGURE 1: Mitochondrial biochemical pathways involved in energy production: including fatty acid oxidation, the respiratory chain, and TCA-cycle (Krebs-cycle) CPT1: Carnitine-Palmitoyltransferase 1; CPT2: Carnitine-Palmitoyltransferase 2; CAT: Carnitine-/AcylcarnitineTransferase; LC: Long-chain; TCA: Tricarboxylic acid cycle.

acid uptake and oxidation (Figure 2). Although these effects have primarily been shown in liver and heart, there is also evidence for a regulatory function of malonyl-CoA in skeletal muscle [4]. Malonyl-CoA levels decrease when the muscle is fuel-deprived or energy consumption is increased during contraction. Long-chain fatty acids cannot freely cross the inner mitochondrial membrane and are shuttled via the carnitine system; acyl-CoA molecules are first coupled to carnitine, catalysed by carnitine-palmitoyltransferase I (CPT 1), and the acylcarnitine complex then crosses the highly impermeable inner mitochondrial membrane. In the mitochondrial matrix space, acylcarnitines are reconverted to acyl-CoA via carnitine-palmitoyltransferase 2 (CPT2) (Figure 1) and subsequently enter beta-oxidation [5]. AcetylCoA derived from beta-oxidation, amino acid-, or glucosemetabolism is channelled into the citric acid cycle. In the citric acid cycle, redox equivalents $\left(\mathrm{FADH}_{2}, \mathrm{NADH}\right)$ are produced which are substrates of the mitochondrial respiratory chain (Figures 1 and 2). Electrons released from these redox equivalents enter the respiratory chain and are finally transferred to oxygen. Electron transport is linked to the build-up of an electrochemical proton gradient across the inner mitochondrial membrane [6]. The electrochemical gradient can be used to synthesize ATP via the mitochondrial ATPsynthase (complex V) or drive transmembrane transport processes directly.

Creatine is phosphorylated by creatinekinase to creatinephosphate (CP) which can be used as an "energy buffer" and is split to ATP via reversal of the creatinekinase-reaction. CP-stores in muscle are only a short-term "energy buffer" and are used-up within the first 30 seconds of exercise.

As mentioned above, energy demand is highly variable in muscle. Energy supply has to be commensurated with energy demand; therefore energy demand is highly regulated.
Calcium (which mediates mechanical muscle contraction) serves as an important regulatory element of metabolism. Regulation of energy metabolism can occur at several levels.

(1) The key enzyme in aerobic mitochondrial energy production via oxidative phosphorylation is ATPsynthase (complex V). Classically, it was believed that ATPsynthase is regulated passively by its substrate ADP; mechanical activation of muscle results in breakdown of ATP to ADP, hence higher substrate saturation of the ATPsynthase and increased flux. We and others have shown in various tissues that the ATPsynthase is actively regulated [7-11], probably via the calcium binding inhibitor protein $(\mathrm{CaBI})$ [12]. This regulatory protein dissociates from the ATPsynthase in response to elevated intramitochondrial calcium concentration during contraction, thus leading to desinhibition of the ATPsynthase, hence higher flux. In animal models, we have previously shown that this regulation breaks down under different pathophysiological conditions [7, 8, 13-16].

(2) Similarly to ATPsynthase, enzymes of the citric acid cycle have been shown to be upregulated in response to increased intramitochondrial calcium levels [17, 18]. Thus, intramitochondrial concentrations of redox equivalents $\left(\mathrm{NADH}, \mathrm{FADH}_{2}\right)$ as substrates of the mitochondrial respiratory chain rise in concert with the activity of mitochondrial ATPsynthase (cf. 1).

(3) Pyruvatedehyrogenase occurs in a nonphosphorylated (active) and a phosphorylated (inactive) form. It is regulated by calcium [19]: muscle contraction is accompanied by increased muscle calcium 


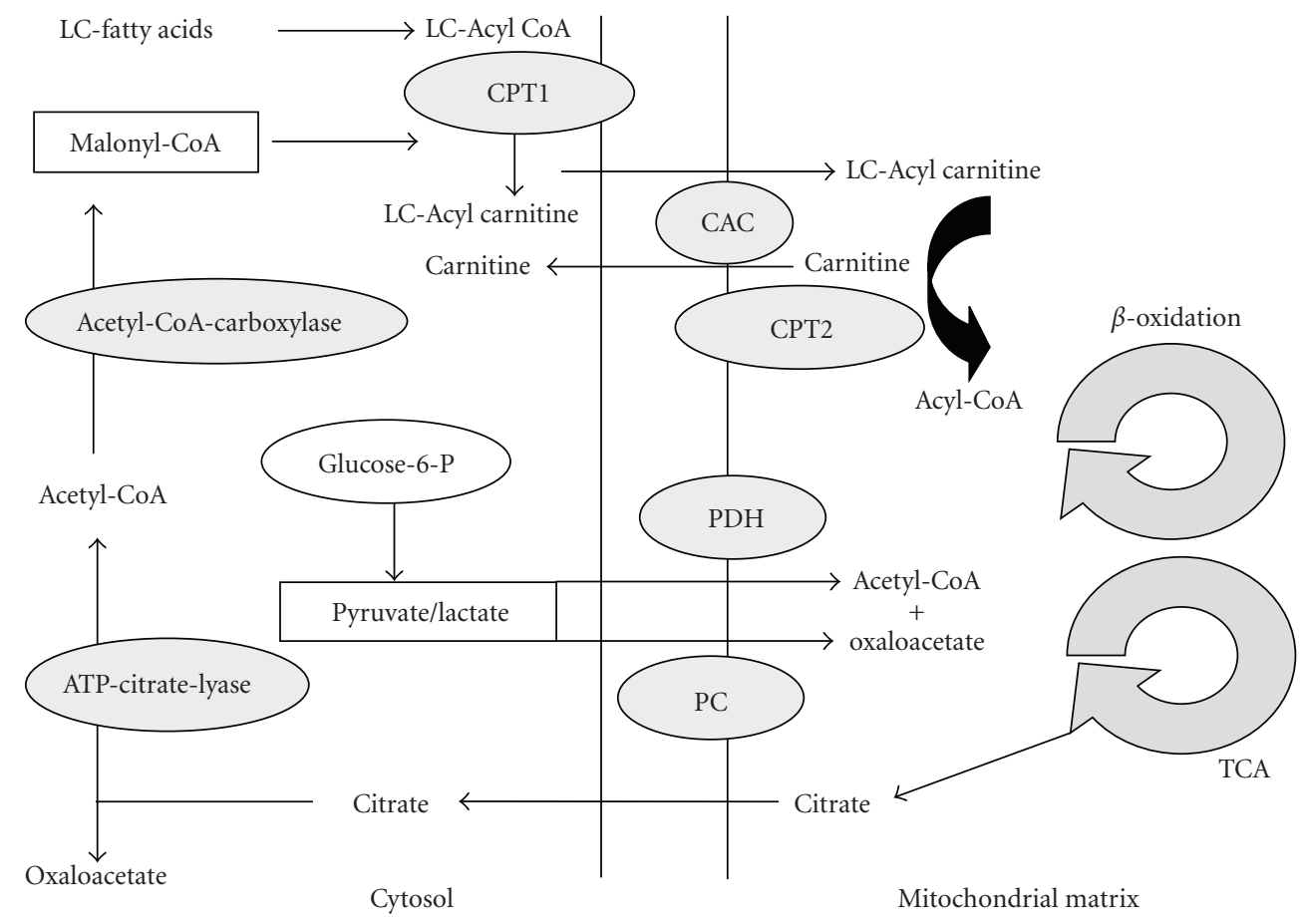

FIGURE 2: llustration of biochemical interactions: the carnitine shuttle, fatty acid oxidation, and glycolysis. The mitochondrial membrane is not permeable to long-chain fatty acids. The carnitine shuttle has to be used to transfer long-chain fatty acids to the mitochondrial matrix space. Once in the mitochondrial matrix space, long-chain acylcarnitines are converted back to long-chain acyl-coenzyme A derivatives and free carnitine via CPT2. Free carnitine is released and the acyl-coenzyme A derivatives will enter the beta-oxidation pathway. CPT1: Carnitine-Palmitoyltransferase 1; CPT2: Carnitine-Palmitoyltransferase 2; CAT: Carnitine-/Acylcarnitine-Transferase; LC: Long-chain; TCA: Tricarboxylic acid cycle; PC: Pyruvate Carcoxylase; PDH: Pyruvate Dehydrogenase.

concentrations which favours the nonphosphorylated form of pyruvatedehydrogenase by activating pyruvatedehydrogenase-phosphorylase and inhibiting kinase [20]. During exercise, muscle glycogenolysis leads to increased production of pyruvate. Pyruvate as the substrate of pyruvatedehydrogenase may subsequently lead to higher substrate saturation, hence higher flux. However, this mechanism is only of minor importance in human skeletal muscle in vivo [20].

Nitric oxide (NO) and its derivatives inhibit mitochondrial respiration by a variety of means. NO can inhibit mitochondrial cytochrome c-oxidase (complex IV) of the respiratory chain by reversibly competing with oxygen for the binding site. Higher concentrations of $\mathrm{NO}$ have been shown to uncouple mitochondria, inhibit complex I, and lead to permeability transition $[21,22]$. Mitochondrial NO synthase may be of particular importance in this context as it is regulated by the mitochondrial membrane potential with reduced membrane potential leading to NO synthase inhibition [23, 24].

Specialized skeletal muscle fibres exist: red muscle fibres are rich in mitochondria and are used for slow and longlasting contractions (endurance) while white muscle fibres predominantly rely on anaerobic glycolysis and can be found in 'fast and short twitch' muscle.
In contrast to skeletal muscle, energy metabolism in heart muscle is almost exclusively aerobic under physiological circumstances. Fatty acids are the main source of energy in the heart: under normal conditions up to $70 \%$ of the energy requirement of the heart is provided by fatty acid oxidation, which may be even higher under certain conditions. The regulatory elements described above for skeletal muscle are operative in cardiac muscle as well.

\section{General Diagnostic Tools in Metabolic Myopathies}

Here we give a general overview of diagnostic procedures which may be useful in the workup of metabolic myopathies. For more specific methods the reader is referred to the special disease section.

At the metabolite level, elevated lactate concentrations in blood and/or urine may be a clue to impaired aerobic energy metabolism (oxidative phosphorylation) and (compensatorily) increased rates of anaerobic glycolysis with lactate as the end product (Pasteur-effect). The lactate/pyruvate ratio in blood reflects the cytosolic NADH/NAD-ratio and may therefore be elevated when aerobic energy metabolism is compromised (e.g., defects of respiratory chain enzymes). The ketone-body ratio ( $\beta$-hydroxybutyrate/acetoacetate, $\beta \mathrm{HOB} / \mathrm{AcAc}$ ) reflects the intramitochondrial NADH/NADratio and may be elevated under certain conditions of 
compromised aerobic energy production (e.g., respiratory chain defects) as well. Impaired oxidative phosphorylation may also be accompanied by elevated excretion of citric acid cycle intermediates (e.g., aconitate, succinate) which can be determined by analysing organic acids in spot-urine. Dicarboxylic acid excretion in urine is a metabolic hallmark both of defects in mitochondrial fatty acid oxidation and respiratory chain enzymes (leading to secondary inhibition of fatty acid oxidation). This finding might also be the result of a diet rich in medium chain triglycerides (MCT enriched diet as used in preterm infants and cholestatic hepatopathy).

Acylcarnitine profiles measured from dried blood spots using the tandem-mass spectrometry technique (tandemMS) may be indicative of fatty acid oxidation disorders. They are now integrative part of the routine newborn screening programme in many countries as a strategy of primary prevention.

A lactate-ischaemia test may be indicated when glycogen storage diseases of muscle are suspected. In healthy individuals, forearm exercise leads to a rapid increase in lactate levels, paralleled by an increase in blood ammonia concentrations. In glycogen storage disease type $\mathrm{V}$ there is no or only attenuated lactate elevation in response to testing, while ammonia production is increased [25]. The nonischaemic forearm exercise test has been found to be less specific and sensitive and is therefore not recommended [26].

For morphological studies of muscle, ultrasound scans, MRI, and biopsy with subsequent histological work-up (including staining) as well as electron microscopy can be used. Traditionally, freeze clamping of percutaneous needle biopsy material has been used to biochemically study muscle energy metabolism. This method has limitations as far as stability of biochemical compounds and repetitive sampling in order to calculate kinetics are concerned.

31-phosphorous $\left({ }^{31} \mathrm{P}\right)$ magnetic resonance spectroscopy (MRS) is an elegant, noninvasive method to judge energy metabolism in vivo [27]. Several peaks corresponding to creatinephosphate (CP), inorganic phosphate, the 3 phosphate groups of ATP, and phosphomonesters can be observed. Kinetic studies using this technique may reveal delayed recovery of phosphocreatine $[28,29]$. Recently, the use of ${ }^{13} \mathrm{C}$-labeled substrates has been advocated to assess mitochondrial metabolism [30].

Another noninvasive technique to assess metabolic function in muscle is positron emission tomography (PET) with glucose as substrate [31]. ${ }^{18} \mathrm{~F}$-Deoxyglucose is commonly used as a marker of glucose metabolism. By comparing glucose metabolism after exercise with reference values, abnormalities may be detected in patients with defects in energy metabolism.

\section{Inborn Errors of Energy Metabolism in Muscle}

\subsection{Disturbance of Glycogen Metabolism \\ (Glycogen Storage Diseases)}

4.1.1. General Aspects. In this section, we deal with defects of glycogen metabolism. Glycogen storage disease type II-also known as Pompe disease-can lead to dysfunction of skeletal and cardiac muscle. As this disease involves lysosomal storage of glycogen but no abnormality in the biochemical pathways leading to energy generation, we shall not deal with this disease in detail. On the other hand, glycogen storage disease type VII is caused by a defect in the glycolytic pathway, with secondary storage of glycogen. As energy metabolism is compromised in this disease we shall describe this entity in detail below.

\subsubsection{Glycogen Storage Disease Type III \\ (M. Cori-Forbes; Deficiency of Amylo-1,6-Glucosidase; Debranching Enzyme)}

Pathogenesis. Glycogen storage disease type III (GSD III; OMIM 232400) is also known as Cori disease, Forbes disease, and limit dextrinosis. The symptoms associated with GSD III were first described in 1952 by Illingworth and Cori and were studied clinically by Forbes, hence the eponyms for this disorder. It is an autosomal recessive disease affecting glycogen metabolism. The overall incidence of this disease is approximately $1: 100,000$ live births in the USA. However, in other populations GSD III may occur with a higher frequency; for example, in the Faroe Islands a prevalence of $1: 3,600$ has been estimated [32].

GSD III is caused by deficiency of the glycogen debranching enzyme amylo-1,6-glucosidase, 4-alphaglucanotransferase (AGL). It degrades glycogen branches releasing glucose in a two-step reaction. Debranching enzyme has two independent catalytic activities: oligo-1,41,4-glucantransferase and amylo-1,6-glucosidase, localised in two distinct protein regions. Patients with debrancher deficiency are classified into four types: IIIa: lack of both glucosidase and transferase activity in liver and muscle; IIIb: lack of both activities in liver only; IIIc: selective loss of glucosidase activity; and IIId: selective loss of transferase activity [33-35].

Most GSD III patients (about 85\%) have AGL deficiency, expressed in both liver and muscle (type IIIa), but some only in liver but not muscle (type IIIb). Differences in tissue expression of the deficient enzyme have been described (immunoreactive material and enzyme activity) [34].

Debranching enzyme is encoded by the $85-\mathrm{kb}$ AGL gene on chromosome 1p21. Genetic screening led to the observation that GSD IIIb patients had mutations in exon 3, whereas GSD IIIa patients had downstream mutations. To date, no common mutation has been described among GSD III patients, except for three alleles, two linked specifically with GSD IIIb, and the third found only in North African Jews with GSD IIIa.

Clinical Features. Clinically, patients with GSD III present in infancy or early childhood with hepatomegaly, hypoglycaemia, and growth retardation. Initially, the clinical picture may be quite similar to GSD I, however biochemically lactate and uric acid concentrations are usually normal in GSD III, and ketone bodies can be found in hypoglycaemia. During puberty and early adulthood, symptoms seem to regress and most patients have only minimal signs of hepatopathy 
$[36,37]$. There is, however, a risk for cirrhosis and in rare cases even hepatocellular carcinoma may develop in the long run [38]. Muscle weakness in those with GSD IIIa is usually minimal in childhood but can become more severe in adulthood; however the clinical course of myopathy is highly variable $[39,40]$. The cause of muscle damage is not clear. Lactate-which could be used as an alternative fuel during exercise-does not increase in GSD IIIa patients (David Weinstein, personal communication) which may explain muscle damage.

The predominant myopathic symptoms in adults are distal weakness (affecting mostly calves and peroneal muscles) and proximal weakness of variable degree with a slow disease progression [40]. Back pain and fatigue may be present. Exercise-induced myoglobinuria is common beyond childhood. Many patients show elevated serum creatine kinase levels. Neuropathy may occur due to glycogen storage in Schwann cells and axons which clinically manifests as polyneuropathy [41].

Osteoporosis is another common symptom in GSD IIIa affecting nearly all patients. Its etiology is multifactorial [42].

Some patients have severe cardiac hypertrophy leading to compromised heart function and even death. Also dilatative cardiomyopathy of the left ventricle was described in anecdotal cases [43]. Heart disease may manifest as ventricular tachycardia as well [44]. Most patients with GSD IIIa do not develop symptoms of cardiomyopathy [45].

Diagnosis. For an accurate diagnosis, biochemical tests with glycogen quantitation and determination of amylo-1,6glucosidase activity in a liver biopsy specimen or erythrocytes are required. Mutation analysis of the AGL gene may be helpful in some cases $[46,47]$ and has been advocated as a screening test using a commercially available test kit [48]. In our opinion, primary screening by mutation analysis is inappropriate. A molecular diagnostic scheme has been proposed to diagnose GSD III noninvasively.

Most of the mutations (more than 30 are known) are nonsense mutations caused by a nucleotide substitution or small insertion or deletion. Some important genotypephenotype correlations have emerged. In particular a specific correlation of exon 3 mutations (17delAG and Q6X) with GSD IIIb has been observed. Three other mutations seem to have some phenotype correlation. Prenatal diagnosis is possible at enzymatic and genetic level [46].

Therapy. Curative therapy is not available for debranching enzyme deficiency. In order to avoid fasting hypoglycaemia in infancy, dietary measures have been proposed. Frequent daytime high protein feedings (45\% carbohydrate, $25 \%$ protein, $30 \%$ fat) and supplementation of uncooked corn starch before sleep proved to be effective in young patients with regard to metabolic control (hypoglycaemia) and growth retardation.

Effects of dietary measures on myopathic symptoms in adults are less well established even though there are reports of improvement in patients following a high-protein diet. In GSD III, gluconeogenesis is intact and protein may be used as a precursor for glucose. Over-treatment with cornstarch can cause deposition of glycogen, which cannot be broken down, leading to accumulation.

In patients with cardiomyopathy, symptomatic pharmacological treatment is mostly used. A diet providing $30 \%$ of energy from protein and avoidance of over-treatment with carbohydrate could be found to stabilize and even reverse cardiomyopathy [49].

\subsubsection{Glycogen Storage Disease Type V (McArdle Disease; Deficiency of Muscle Phosphorylase)}

Pathophysiology. Myophosphorylase activity is deficient in McArdle disease (OMIM 232600); the disorder is inherited as an autosomal recessive trait. The myophosphorylase gene is located on chromosome 11q13 and spans 20 exons [50]. The most common mutation in Northern Europe and North America is the nonsense mutation at R50X, previously referred to as R49X [51, 52]. Genotype-phenotype correlation is poor, modifying genes as angiotensin converting enzyme or actin 3 genes may play a role [53-55].

As a consequence of myophosphorylase deficiency, the contracting muscle is not able to mobilize adequate amounts of muscle glycogen during the initial anaerobic phase of exercise. This leads to an energy deficit which cannot be covered by creatinephosphate stores. During the aerobic phase of exercise, blood-born substrates like fatty acids and amino acids as well as glucose can be used as energy substrates. However, the capacity of the tricarboxylic acid cycle (TCA- or Krebs-cycle) is compromised as well. This is due to the lack of pyruvate which is normally generated from glucose via glycolysis and has an anaplerotic effect on the TCA-cycle via oxaloacetate. We could show adaptive processes at the level of mitochondrial ATPsynthase in a patient with McArdle disease (poster presentation at the 4th World Muscle society Congress 1999: Das AM, Kohlschütter A; Upregulation of mitochondrial respiratory chain enzymes in GSD V).

Clinical Features. Symptoms typically begin in the second to third decade of life. As first reported by McArdle in 1951, patients typically suffer from exercise-induced muscle pain, cramps, and fatigue [56]. These symptoms are most pronounced during the first few minutes of exercise (anaerobic phase) and may be accompanied by rhabdomyolysis leading to myoglobinuria with burgundy discoloration of urine. Acute renal failure secondary to myoglobinuria has been reported in a few patients. After blood supply to the exercising muscle has increased, symptoms disappear as energy supply is reinstored; this phenomenon is known as "second wind". Most patients with McArdle disease learn to cope with their problems by resting until muscle pain subsides and resuming activity as soon as the aerobic phase sets in (oxidizing fatty acids and other blood-born substrates).

A less common manifestation of McArdle disease is permanent muscle weakness. This kind of symptom usually occurs later in life. Some patients may have proximal and symmetric weakness while others have asymmetric weakness mimicking facio-scapulohumeral dystrophy (FSHD) [57]. 
Diagnosis. Plasma creatinekinase activity is usually elevated at rest and rises in response to muscular activity. Patients fail to produce lactate during the ischaemic forearm test while ammonia concentration increases. Inosine, hypoxanthine, and uric acid also increase reflecting accelerated recycling of purine nucleotides in the face of insufficient ATPproduction.

Muscle biopsy specimen usually shows subsarcolemmal and intermyofibrillar vacuoles filled with glycogen. Ultrastructurally, there are deposits of normal-looking free glycogen particles and occasional alterations of sarcoplasmic reticulum and mitochondria. Staining for phosphorylase is negative in muscle fibres whereas smooth muscle of blood vessels stains normally.

Biochemically, reduced activity of phosphorylase may be found by enzymatic assays. Diagnosis may be further corroborated by mutation analysis (see above). This allows definitive prenatal testing where appropriate.

Lack of lactate formation may lead to absent acidification of myoplasm as judged by ${ }^{31} \mathrm{P}-\mathrm{MRS}$ [58]. The phosphocreatine/ATP-ratio is usually elevated at rest, phosphocreatine depletion during exercise is larger than normal, and recovery rate of phosphocreatine after exercise is slower $[58,59]$. Changes in T2-relaxation are often absent in exercising muscle from McArdle patients [60]. EMG is usually unrevealing between acute attacks.

Treatment. Both pharmacological and dietary interventions have been tried in order to improve exercise intolerance. Therapy of McArdle disease was subject of a recent Cochrane review [61]. 24 studies were reviewed; 12 of these fulfilled the criteria for inclusion. High protein, high carbohydrate, and high fat diets as well as ketogenic diet have been tried without significant effects. Furthermore, glucagon administration, verapamil, ACE-inhibitors, sodium dantrolene, gentamicin, vitamin B6, and creatine have been tested. Low-dose creatine may have a modest benefit in some patients [62] while higher-doses may lead to deterioration [63]. All other interventions did not have any benefit. The Cochrane review concludes that none of the tested pharmacological or dietary interventions can be recommended.

\subsubsection{Glycogen Storage Disease Type VII (Tarui Disease; Deficiency of Muscle Phosphofructokinase)}

Pathophysiology. Tarui disease (GSD VII; OMIM 232800) is caused by deficiency of muscle phosphofructokinase. It is inherited as an autosomal-recessive trait (gene map locus of muscle phosphofructokinase PFKM 12q13.3).

Phosphofructokinase is a tetrameric enzyme derived from 3 distinct genetic loci that code for muscle, liver, and platelet isoforms. Mammalian PFK is a tetramer made up of various combinations of 3 subunits: muscle (PFKM), liver (PFKL), and platelet (PFKP), the genes for which are located on chromosomes $12 \mathrm{q} 13,21 \mathrm{q} 22$, and $10 \mathrm{p}$, respectively. The composition of the tetramers differs according to the tissue type. Muscle and liver PFK are homotetramers of $4 \mathrm{M}$ and $4 \mathrm{~L}$ subunits, respectively. Erythrocytes contain both L and M subunits.
The muscle phosphofructokinase gene is located on chromosome 12 and about 20 mutations have been described $[64,65]$. Phosphofructokinase is not involved in glycogen metabolism but is a glycolytic enzyme. The PFKM gene encodes the muscle isoform of phosphofructokinase (PFK) (ATP:D-fructose-6-phosphate-1-phosphotransferase). PFK catalyzes the irreversible conversion of fructose-6-phosphate to fructose-1,6-bisphosphate and is a key regulatory enzyme in glycolysis.

Tarui disease has a relatively high prevalence in Japanese people; in the western hemisphere it is prevalent in patients of Jewish-Russian extraction.

If the muscle isoenzyme of phosphofructokinase (PFKM) is deficient, not only is glycolysis in muscle affected but also glycolysis in erythrocytes is reduced to about 50\% (as the liver isoenzyme is fully active). As erythrocytes have no mitochondria, this relatively mild reduction of glycolytic flux can critically reduce energy supply resulting in haemolysis.

Clinical Features. Clinical symptoms are very similar to McArdle disease with exercise-induced pain, muscle cramps, and fatigue. As in McArdle disease the initial anaerobic phase of exercise is affected when the muscle has to rely on anaerobic glycolysis which is defective in Tarui disease. The resulting energy shortage leads to the clinical symptoms as first described by Tarui et al. in 1965 [66]. There are some features which may differentiate McArdle disease from Tarui disease: in Tarui disease, symptoms are more severe and often already present during childhood, often accompanied by nausea and vomiting as well as compensated haemolytic anaemia, hyperuricaemia, and an abnormal polysaccharide which is periodic acid-Schiff positive. This polysaccharide has a fine fibrillar appearance resembling amylopectine.

After carbohydrate-rich meals, exercise intolerance is often exacerbated. The pathophysiological basis for this observation is probably the inability of muscle to use glucose (anaerobically and aerobically) on one hand and hyperinsulinism caused by hyperglycaemia with subsequent lowering of fatty acids as energy substrates for muscle on the other hand.

As in McArdle disease, there are variant forms of the disease. One variant leads to muscle weakness in later adult life while the other leads to hypotonia during infancy with subsequent progressive myopathy leading to early death in younger children.

Diagnosis. As in glycogen storage disease type $\mathrm{V}$ the ischaemic forearm test has a pathologic result with insufficient lactate production accompanied by a rise in ammonia levels.

Histochemical and biochemical demonstration of the enzyme defect proves the suspected diagnosis. Mutation analysis may be added for further confirmation.

${ }^{31} \mathrm{P}-\mathrm{MRS}$ shows increased phosphocreatine/ATP ratio in resting muscle, ATP depletion, larger than normal phosphocreatine depletion during exercise with slow recovery rate and absence of myoplasmic acidification $[67,68]$. 
Therapy. There is no specific therapy for this condition. Most patients try to avoid strenuous exercise, by slowing down speed or pausing after onset of symptoms. Pain and fatigue can be reduced, after adequate blood (and fatty acid) supply sets in. Physical activity may be resumed without problems after a short break.

\subsection{Disturbance of Fatty Acid Oxidation}

\subsubsection{General Aspects}

General Aspects of Pathophysiology in FAO Disorders (FAODs). Mitochondrial fatty acid oxidation represents an important pathway for energy supply in skeletal muscle during both quiescence and periods of physical exercise. Cardiac muscle preferentially oxidizes fatty acids for energy generation and may have a limited ability to rely completely on glucose during periods of stress $[4,69]$.

Among metabolic myopathies, long-chain mitochondrial fatty acid oxidation defects (FAODs) are probably the most difficult to identify due to the episodic character of clinical and biochemical abnormalities which may completely disappear during anabolism. The pathway of fatty acid beta-oxidation includes at least 25 enzymes and specific transport proteins. Deficiencies in more than 50 percent of these enzymes are known to cause disease in humans. Apart from overall energy deficit in the muscle toxic (lipophilic) compounds resulting from compromised fatty acid oxidation, such as long-chainacylcarnitines, CoA-esters, or their free long-chain fatty acids may play a role in the pathogenesis of clinical manifestations.

In this section we will focus on the following more common disorders: Carnitinepalmitoyltransferase 2 (CPT2), mitochondrial trifunctional protein (MTP), very-longchain-acyl-CoA dehydrogenase (VLCAD), multiple acylCoA (MAD), as well as primary carnitine/carnitine transporter-deficiency (CTD).

General Clinical Aspects of Long-Chain FAOD. In disorders of long-chain fatty acid oxidation, clinical symptoms are usually triggered by common infections, prolonged exercise, and exposure to cold and prolonged fasting (catabolism). In long-chain FAOD, the phenotype ranges from benign forms with isolated skeletal muscle involvement to severe phenotypes leading to early death as has been initially described in patients with carnitine palmitoyltransferase 2 (CPT2) deficiency [70-72].

Newborns and infants commonly present with a multisystemic manifestation (including liver, muscle, and brain involvement) often triggered by physiological catabolism soon after birth, whereas onset later in life usually presents with exercise intolerance, muscle weakness, and sometimes myoglobinuria during metabolic decompensation. Myoglobinuria may result in acute renal failure if fluid intake is restricted. Long-chain FAOD-associated myopathies of the more chronic type manifest predominately during aerobic endurance-type activity or under metabolically stressful conditions. In contrast to patients with glycogen storage diseases of muscle, patients suffering from FAOD do not experience a "second-wind" phenomenon.

General Diagnostic Procedures in FAOD. The diagnosis is based on detecting the accumulation of specific biochemical markers such as acylcarnitine metabolites in blood, dicarboxylic acids, and acylglycines in urine by routine newbornor selective screening.

Medium- and long-chain fatty acid oxidation defects have been included in the routine neonatal screening program, for example, by the German screening commission. Newborn screening for FAOD promises to identify many affected patients before the onset of symptoms or in the early phase of metabolic decompensation. Physiological catabolism in the first days of life is helpful in demasking biochemical abnormalities at metabolite level. Confirmation of suspected mitochondrial fatty acid oxidation defects based on an initial abnormal newborn- or selective metabolicscreening by tandem mass spectrometry should include enzyme and whenever possible molecular analyses. Biochemical testing of urine (organic acids) may be unrevealing. Patients who are not aware of their defect could be easily at risk of decompensation when exposed to different triggers, that is, prolonged fasting, fever, or strenuous physical exercise. Therefore, neonates (and older patients) suspected to suffer from an FAOD should receive special monitoring and follow an emergency regime until the metabolic defect is excluded.

Pathologic findings in muscle biopsies are most often nonspecific. Moderate lipid storage is present in only some cases.

Of particular importance is the possibility of secondary respiratory chain dysfunction in patients with FAO defects or vice versa [73].

Diagnostic procedures in long-chain FAO disorders have recently been summarized based on the results of a consensus workshop [74].

Specific diagnostic options are mentioned in more detail below, where appropriate.

General Treatment Principles of FAOD. Evidence-based treatment recommendations for long-chain FAO defects are not available [75]. However, at national/multinational levels consensus protocols exist [76]. Current management of patients with long chain fatty acid oxidation defects includes long-term dietary therapy including avoidance of fasting, low fat diet with the restriction of long-chain fatty acid intake, and substitution with medium chain fatty acids. The longterm outcome of patients treated by dietary modification remains unknown $[74,76]$. Dietary long-chain fat restriction depends on the severity of the underlying enzyme defect.

Episodic muscular symptoms such as rhabdomyolysis are pathogenetically attributed to energy deficiency, and symptoms are reversed or may be prevented by sufficient energy supply in the form of carbohydrates or MCT [77]. The incidence of overweight and obesity is increasing among children with long-chain FAOD. A diet higher in protein and lower in carbohydrate may help to lower total energy intake while maintaining good metabolic control [78]. 
In the past, supplementation with carnitine was proposed in fatty acid oxidation defects for detoxification of accumulating long-chain acyl-CoA esters. Actually, carnitine supplementation in long-chain FAOD is not recommended [74].

Further therapeutic options have anecdotally been described in single cases: Triheptanoin (seven-carbon medium chain fatty acid) supplementation could show an improvement of cardiac and muscular symptoms in patients suffering from long-chain fatty acid oxidation disorders [79]. PPARalpha agonists (like fibrates) stimulated mitochondrial fatty acid oxidation in vivo in both liver and muscle and might be a therapeutic option in inborn errors of FAO [80-82].

D,L-3-hydroxybutyrate could be a therapeutic option for cerebral and cardiac complications in severe FAO [83].

Disease-specific therapeutic options are discussed below.

\subsubsection{Carnitine Palmitoyltransferase 2 (CPT2) Deficiency}

Pathophysiology. CPT2 is located at the inner aspect of the inner mitochondrial membrane catalyzing the conversion of long-chain acyl-carnitines to acyl-CoA esters. CPT2 deficiency (OMIM 600650) is inherited as an autosomal recessive trait resulting in the accumulation of long-chain acylcarnitines. The gene is located on chromosome 1 p32 [84]. There are three phenotypes of CPT2 deficiency: the "classic muscular form" (OMIM 255110) is most frequent and shows onset in childhood or adulthood with exerciseinduced muscle weakness and rhabdomyolysis. CK levels are found to be elevated during symptomatic periods, and carnitine levels are usually normal [70]. The prevalent c.338C $>$ T (p.Ser113Leu) mutation is found in about $60 \%-$ $75 \%$ of mutant alleles [85]. This CPT2 mutation is thermolabile resulting in the degradation of the protein during fever or muscular exercise accompanied by elevated body temperature [86].

Clinical Features. A "severe neonatal form" (OMIM 608836) presents in the newborn period with nonketotic hypoglycemia, cardiomyopathy, muscle weakness, and renal dysgenesis in some patients [87]. Most of these patients die within days after birth [88]. The "infantile multisystemic phenotype" (OMIM 600649) is often fatal in the period from 3 to 18 months of age. It presents with seizures, hepatomegaly, nonketotic hypoglycemia, cardiomyopathy, and muscle weakness. Plasma free carnitine levels are low and long-chain acylcarnitines high [89]. These different clinical presentations appear to be correlated with different residual CPT2- and overall long-chain FAO-capacity [90].

Muscle CPT2 deficiency clinically manifests in patients harboring only a single heterozygous mutation [91]. Compound heterozygosity for a mild and a severe mutation can be associated with either the mild muscle or the severe multisystemic infantile form [92]. Several homozygous mutations have been reported, associated with either a prominent skeletal muscle involvement or with a severe generalized phenotype [93, 94]. Severe and intermediate phenotypes were found to correlate with biochemical indices and genetic analysis. Milder phenotypes suggest little evidence for genotype-phenotype correlation [86].

The presence of only one severe mutation (the other being a milder one) in a CPT2 deficient patient might be sufficient to lead to life-threatening events [92]. Severely compromised long-chain fatty acid oxidation may point to a more severe course $[90,95]$.

\subsubsection{The Mitochondrial Trifunctional Protein (MTP) Deficiency}

Pathophysiology. MTP is an enzyme complex involved in the beta-oxidation of fatty acids with chain lengths of C12 to C18. It catalyzes three reactions in the oxidation of long chain fatty acids and consists of the following enzymes: 3-hydroxyacyl-CoA dehydrogenase (LCHAD), 2-enoyl-CoA hydratase, and 3-ketoacyl-CoA thiolase.

MTP is a hetero-octamer of 4 alpha- (OMIM 600890; with LCHAD and 2-enoyl-CoA hydratase activities) and 4 beta-subunits (OMIM 143450; with 3-ketoacyl-CoA thiolase activity). The alpha- and beta-subunits are encoded by different nuclear genes, consisting of 20 and 16 exons, respectively. Both are located on chromosome 2p23 [96].

A deficiency in this heteromeric complex (OMIM 609015) was first described in 1992 [97]. In contrast to the large number of isolated LCHAD-deficient patients (OMIM 609016), only few MTP alpha- and beta-subunitdeficient patients were published [98]. Molecular studies in MTP-deficient patients show a wide range of "private" mutations in both the alpha- and beta-subunit, in contrast to the common $1528 \mathrm{G}>\mathrm{C}$ mutation (E474Q) in LCHAD deficiency. Thiolase expression levels and the different rates of thiolase degradation as a result of either alpha- or beta-subunit mutations were found to correlate well with the severity of clinical manifestations [96].

The biochemical hallmarks of this disorder are the accumulation of long-chain 3-hydroxyacylcarnitines and free fatty acids in blood as well as dicarboxylic acids in urine.

Clinical Features. These long-chain FAO defects have variable presentations. They may present in the neonate or infant with sudden death, hepatopathy (Reye-like disease), hypoketotic hypoglycaemia, rhabdomyolysis, myopathy, cardiomyopathy, and capillary leak syndrome often associated with lung oedema. Late complications such as peripheral neuropathy, pigmentary retinopathy, retinal degeneration, and progressive visual loss occur [97-100]. The clinical spectrum in MTP-deficiency seems to be broader than in isolated LCHAD-deficiency, usually with a first manifestation earlier in life with a lethal outcome.

Three phenotypes have been reported in patients with alpha- or beta-subunit mutations: a "lethal form" with predominating cardiac involvement, an "infancy-onset" hepatic presentation, and a milder "late-onset" neuromyopathic form [96]. The main features of the neuromyopathic form with late-onset are progressive peripheral neuropathy and episodic, often exercise-induced myoglobinuria. 


\subsubsection{Very Long-Chain Acyl-CoA Dehydrogenase (VLCAD) Deficiency}

Pathophysiology. VLCAD is bound to the inner mitochondrial membrane and catalyses the first step of the long-chain fatty acid beta oxidation spiral [101]. VLCAD deficiency (OMIM 201475) is inherited as an autosomal recessive trait (gene map locus 17p13).

Clinical Features. According to the age of onset, three phenotypes have been described [102-105]: a severe "infantile form" presenting in the neonatal period with hypertrophic cardiomyopathy and liver failure, a "childhood-onset" type with hypoketotic hypoglycaemia, and a "juvenile or adultonset" muscular form characterized by recurrent episodes of rhabdomyolysis triggered by prolonged exercise or fasting (similar to CPT2 deficiency).

A clear genotype-phenotype correlation could be found [102]: patients with the severe childhood phenotype have mutations that result in absence of enzyme activity, whereas patients with the milder childhood and adult (myopathic) phenotypes have mutations that result in residual enzyme activity. However, a recent report of life-threatening disease with cardiomyopathy and hypoketotic hypoglycaemia in a previously healthy 32 -year-old woman underscores the potential risk of severe complications, even in adults [106].

The milder form of VLCAD deficiency is increasingly recognised due to the more wide-spread use of tandem mass spectrometry, allowing the (presymptomatic) detection of abnormal long-chain acylcarnitines in blood samples during neonatal screening [107]. Some neonates show biochemical abnormalities in routine screening but do not suffer from clinical disease. For this, the term "Non-disease" (which is poorly defined as a condition where late-onset disease is possible) has been coined. Clinical observation of patients with late-onset presentation of VLCAD deficiency is scarce [106, 108-117].

\subsubsection{Multiple Acyl-CoA Dehydrogenase Deficiency (MADD; Also Glutaric Aciduria Type II, GA II)}

Pathophysiology. This is an autosomal recessively inherited disorder of fatty acid, amino acid, and choline metabolism. In multiple acyl-CoA dehydrogenase deficiency (MADD, OMIM 231680) large excretion not only of glutaric acid but also of lactic, ethylmalonic, butyric, isobutyric, 2-methylbutyric, and isovaleric acids in urine occurs. MADD results from deficiency of one of three molecules: the alpha (ETFA) and beta (ETFB) subunits of electron transfer flavoprotein (ETF), and electron transfer flavoprotein dehydrogenase (ETFDH), gene map locus 15q23-q25, 19q13.3, and 4q32qter.

Clinical Features. The clinical picture of MADD due to the different defects appears to be similar. The most severely affected patients have congenital anomalies and die in the newborn period; other patients have hypoglycaemia, encephalopathy, muscle weakness, or cardiomyopathy. Mildly affected patients present with muscle weakness only [118]. Some patients present with respiratory dysfunction [119]. Each of the single enzyme defects can lead to a mild or severe course, depending presumably on the location and nature of the intragenic lesion. Therefore, mutations can result in dysfunction of electron transfer flavoprotein (ETF) or ETF-ubiquinone oxidoreductase (ETF-QO). It results in secondary deficiencies of a variety of FAD-dependent enzymes, including glutaryl-CoA dehydrogenase, as well as acyl-CoA dehydrogenases from FAO and branched-chain amino acid metabolism [120].

Mutations in ETFDH, encoding ETF-QO, have been associated with both riboflavin-responsive and nonresponsive MADD as well as a myopathic form of CoQ 10 deficiency [121]. Pathomechanisms responsible for these different phenotypes are not well defined $[122,123]$. As with VLCAD deficiency, there is a relationship between ETF/ETFDH genotype and phenotype in patients with MADD.

In MADD early diagnosis is essential as some patients respond to pharmacological doses of riboflavin. It has recently been shown that rapid diagnosis of MADD via newborn screening using tandem-MS is possible. Only in some of these children, disease causing mutations could be found; some were responsive to riboflavin even without a mutation (presented as an abstract at 11th ICIEMcongress in San Diego, 2009: Olsen et al. Multiple AcylCoA Dehydrogenation Deficiency). Furthermore, coenzyme Q10 concentrations should be determined in patients suffering from riboflavin responsive MADD.

Therapeutic Options in MADD. The effect of riboflavin and coenzyme Q10 should be tested [121]. Ketone bodies like D, L-3-hydroxybutyrate are discussed as a therapeutic option [83].

\subsubsection{Systemic Primary Carnitine Deficiency/Carnitine Transporter Deficiency (CTD)}

Pathophysiology of CTD. Carnitine is ubiquitous in nature, especially high in muscle tissue of higher organisms, but low in vegetables. In humans, biosynthesis takes place in liver and kidney. To fulfil its function, intracellular carnitine concentrations have to be rather high, especially in skeletal muscle cells. Therefore, active carnitine transport mechanisms from blood into the cell have to exist. Translocation is mediated by the carnitine transporter, a special protein, which is located in cell membranes. The active transport of carnitine into tissue takes place against a concentration gradient, permitting tissue carnitine concentrations to be 20to 50 -fold higher than plasma levels [124]. Furthermore, this carnitine transporter reabsorbs all carnitine from primary urine into blood. The sequence of the 557 amino acids of the carnitine transporter is encoded on chromosome 5q31.2-32.

In case of carnitine transporter deficiency (OMIM 212120)—inherited as an autosomal recessive traitcarnitine is lost in considerable amounts via urine; the body becomes depleted of carnitine. As a consequence, impaired oxidation of long chain fatty acids leads to deficient energy generation as well as reduced ketogenesis during fasting, and accumulation of lipids in liver and muscle [125]. 
Clinical Features of CTD. Different courses of CTD are known. The most severe presentation can lead to sudden infant death syndrome (SIDS) already in the neonatal period [126]. Mean age of presentation with "Reye-like" manifestations as hypoketotic hypoglycemic encephalopathy is between one month and a maximum of 5 years with an estimated median of 1.5 years [127].

The metabolic deterioration is usually triggered by prolonged fasting, reduced oral intake, or vomiting during viral illness. Those who survive critical illness suffer from recurrent episodes of encephalopathy if the defect is not diagnosed and no carnitine supplementation is given [128]. Later in life, the disorder can present with skeletal muscle disease causing muscular hypotonia with mild motor delay.

Progressive cardiomyopathy in late infancy or early childhood (usually between 1 and 7 years of age, median 3 years) leads to dilatative cardiomyopathy with greatly reduced left ventricular ejection fraction, abnormal T-waves, and signs of ventricular hypertrophy $[128,129]$. Bradycardia and atrial arrhythmias are possible [130]. Sometimes no overt cardiomyopathy, but only mild interventricular septum hypertrophy, does occur [130]. If no carnitine replacement is given, progressive congestive heart failure may lead to death. Another form of cardiac deterioration was first described in 2004. A 15-year-old girl was diagnosed with cardiac arrest due to ventricular fibrillation without overt cardiomyopathy. ECG showed nonsustained ventricular tachycardia with periods of sinus rhythm and ventricular premature beats, while echocardiography revealed normal left ventricular dimensions and a borderline left ventricular hypertrophy with good contractility [131].

Nicola Longo (personal communication) stated that many of the patients who die suddenly with primary carnitine deficiency do not have cardiomyopathy (beyond childhood), but "only" sudden arrhythmias. The mechanism causing the arrhythmias is subject to ongoing studies. Adultonset lipid storage myopathy presenting with myalgia and muscular fatigue during prolonged exercise could be shown to be caused by primary carnitine deficiency. Symptoms and abnormalities in muscle biopsy improved tremendously after four months of oral therapy with carnitine supplementation [132].

Asymptomatic courses are known as well: Spiekerkoetter et al. reported a 28-year-old man diagnosed with CTD, based on a positive family history with disease manifestation in one of his sons [133]. Four asymptomatic mothers were discovered to have CTD when being examined for low carnitine found by neonatal screening in blood spots of their offspring [134].

Patients with identical mutations can have different age of onset and different type of clinical presentation [135]. Frequency of the different courses is unknown. Many of the adults dying from ventricular fibrillation may have suffered from undiagnosed CTD. We are aware of at least 4 young adults from the Faroe Islands who showed this course (unpublished data).

Diagnostic Options in CTD. Laboratory hallmarks of CTD are very low free plasma carnitine concentrations (lower than
$10 \mu \mathrm{mol} / \mathrm{L}$ ) and increased fractional excretion of carnitine in urine. During decompensation, glucose and ketone bodies are inappropriately low. Transaminases and ammonia may be moderately elevated, and metabolic acidosis, prolonged prothrombin time, and elevated CK do occur.

Final confirmation of the diagnosis can be done by showing low activity of the transporter with decreased carnitine uptake in fibroblasts. Mutation analysis can be performed.

Therapeutic Options in CTD. The treatment of CTD is easy and without severe adverse reaction apart from a strange "fish-like" body odour. Carnitine is supplemented in a dose of 50-200 mg per $\mathrm{kg}$ body weight and day given in at least three portions.

\subsection{Disturbance of Respiratory Chain Function}

Pathophysiology. The respiratory chain is responsible for the aerobic energy generation from different substrates like fatty acids, glucose, and amino acids. It is integrated in the inner mitochondrial membrane which is almost impermeable. Electrons from the reduction equivalents $\mathrm{NADH}$ and $\mathrm{FADH}_{2}$ enter the respiratory chain via complex I and complex II, respectively. Electrons then follow a potential gradient and are finally transferred to oxygen. Flow of electrons leads to the generation of an electrochemical proton gradient across the inner mitochondrial membrane as protons are pumped out of the mitochondrial matrix [6]. This gradient can be directly used, for example, for the transport of charged substances (e.g., ATP-ADP exchange). On the other hand it can be used to drive the ATPsynthase (complex V) reaction which dissipates the proton gradient across the inner mitochondrial membrane. The mitochondrial ATPsynthase has been shown to be actively regulated both in heart [136] and in skeletal muscle [137].

The mitochondrial genome consists of 16,569 base pairs (http://www.mitomap.org/MITOMAP). Mitochondria have their own mitochondrial DNA. Some 80 mitochondrial proteins are present in the mitochondrium, 13 of these are encoded on the mitochondrial DNA. Some subunits from complexes I, III, IV, and V are encoded on the mitochondrial DNA while other subunits from complexes I-V are nuclearencoded. As mitochondrial DNA is inherited from the mother, respiratory chain defects due to mutations in the mitochondrial DNA follow a maternal pattern of inheritance. Mitochondrial DNA encodes both tRNA involved in protein synthesis and genes responsible for the generation of subunits of respiratory chain complexes. Nuclear-encoded mutations are inherited as an autosomal-recessive trait.

Most of the cells contain several mitochondria; each mitochondrium harbours up to 10 copies of mitochondrial DNA. Mutations in mitochondrial DNA normally do not affect all DNA of one cell and not all cells of one organ but there is a mixture of healthy and diseased cells (heteroplasmy). The relative distribution between both subsets can vary during life; the course of changes is unpredictable. Deleted DNA may replicate faster due to shortened DNA, leading to a shift towards mutated DNA. 
Once a threshold is reached, clinical symptoms develop (depending on the organ involved). This also explains the high variability of clinical symptoms and lack of genotypephenotype correlation. Highly specialized cells, like mature erythrocytes, do not contain mitochondria and are therefore spared from mitochondrial dysfunction; however immature forms of these cells may be affected.

Mitochondrial dysfunction leads to a lack of energy in aerobic tissues and production of possibly toxic free radicals which finally may induce apoptosis [138, 139]. Mitochondria are not only responsible for energy generation, but are able to take up and release calcium, thus regulating calcium homeostasis. This mitochondrial function is partly lost in respiratory chain defects.

Clinical Features. Based on the central role of energy generation within cells, almost all organs may be affected by respiratory chain disorders. Cellular heteroplasmy leads to a highly variable clinical picture of a single mutation. Organs showing a high rate of aerobic energy metabolism are often compromised first by respiratory chain disorders. Skeletal and heart muscle are frequently affected.

The first description of a mitochondrial myopathy was by Luft et al. [140], in a patient with increased thermogenesis in response to muscle exercise and ultrastructural abnormalities of mitochondria. This patient presumably had "loose coupling" between mitochondrial ATP production on one hand and flux of electrons across the respiratory chain on the other hand [141]. In the late 1980s and early 1990s, more and more patients with mitochondriopathies due to mitochondrial dysfunction were reported.

Skeletal muscle dysfunction mostly presents as exercise intolerance; rarely pain is reported. There may be either isolated myopathy or a more complex disease with multisystemic manifestations.

Cardiac manifestations are limited exercise capacity, overt heart failure in later stages accompanied by lung oedema and hypertrophic cardiomyopathy; sometimes arrhythmias are the dominating feature. Echocardiography shows heart hypertrophy, sometimes dilatative cardiomyopathy, decreased shortening fraction, and often diastolic dysfunction. The latter is caused by incomplete (energydependent) uptake of calcium from the cytosol to the sarcoplasmic reticulum and mitochondria. The ECG may occasionally resemble infarction as this is also accompanied by energy depletion (in the case of infarction due to lack of oxygen).

Frequently, other organs are compromised by mitochondriopathy and nonmuscular features like epilepsy, stroke, and so forth, may predominate. Certain combinations of clinical symptoms have been summarized as syndromes. The terms Kearns-Sayre syndrome, mitochondrial encephalopathy, lactic acidosis, and stroke-like episodes (MELASs), as well as Leber's hereditary optic neuropathy (LHON) were coined and shown to be due to mutations in the mitochondrial DNA. However, this is rarely helpful in the diagnosis or treatment due to the large variability of clinical symptoms in one single mutation.
Diagnosis. Creatinekinase (CK) activity may often be elevated in plasma as an unspecific finding. Lactate concentration in blood-reflecting a compensatory increase in flux of anaerobic glycolysis - may be elevated (normal: $<2.6 \mathrm{mmol} / \mathrm{L}$ ). Lactate/pyruvate ratio reflects cytoplasmic $\mathrm{NADH} / \mathrm{NAD}$ ratio and may be increased (normal: less than 20 ) in defects of respiratory chain complexes I-V. However, if only single organs are affected, lactate levels as well as lactate/pyruvate ratio in blood may be normal. Diagnostic accuracy of the lactate/pyruvate ratio in blood has been shown to increase with higher lactate concentrations [142]. In response to oral carbohydrates, lactate concentration in blood may rise inappropriately in respiratory chain disorders. If cytosolic NADH/NAD levels remain normal as in pyruvatedehydrogenase deficiency, lactate/pyruvate ratio in blood is normal. As many metabolic pathways have an effect on lactate and pyruvate metabolism, lactate/pyruvate ratios may easily fluctuate and may be unreliable. If determined in tissue cultures the reliability of the lactate/pyruvate ratio increases $[143,144])$. The ketone body ratio $(\beta-$ hydroxybutyrate/acetoacetate) reflects the intramitochondrial NADH/NAD ratio and may be increased in respiratory chain defects. Alanine may be elevated in blood.

In urine, increased amounts of lactate, pyruvate, and ketone bodies may be found. Dicarboxylic aciduria reflecting secondary inhibition of fatty acid oxidation (by accumulating NADH and FADH) may be observed. Ethylmalonic acid and 3-methylglutaconic acid excretion provide indirect evidence for mitochondrial dysfunction.

Muscle biopsy is an important diagnostic tool. The number of type I muscle fibres (red) may be compensatorily elevated. "Ragged red" fibres—seen on a modified Gomorri trichrome stain - are the hallmark of mitochondrial disease, however these are not obligatory. Mitochondrial proliferation may also become evident using the succinate dehydrogenase and cytochrome oxidase stains. The classic electron microscopic findings are paracrystalline inclusions, crystals consisting of mitochondrial creatinekinase.

Muscle enzymology is the next biochemical step in the diagnostic work-up. Activities of respiratory chain complexes can be assayed either in a group of 2 enzymes or as single enzyme activities. Citratesynthase activity is usually determined as a mitochondrial marker enzyme. We have shown that the mitochondrial ATPsynthase (complex V) is regulated both in rat heart [136] and in human skeletal muscle [137] in response to energy demand. Active regulation of mitochondrial ATPsynthase requires an intact inner mitochondrial membrane. We therefore advocate measurement of respiratory chain enzymes in fresh muscle preparations, as freezing leads to the disruption of the inner mitochondrial membrane. Using this technique, we have shown that secondary downregulation of ATPsynthase (complex V) occurs in response to reduced electron flow across complexes I-IV [145]. Respiratory chain activity can be influenced by a number of endogenous and exogenous factors. For example, limb immobilisation has been shown to lead to downregulation of mitochondrial pathways [146].

The heteroplasmic nature of most pathogenic mitochondrial DNA mutations makes genetic diagnosis difficult [147]. 
Genotype-phenotype correlation has been reported to be poor [148]. By mutation analysis, mitochondrial depletion syndromes may be identified which are characterized by a reduction in mitochondrial DNA copy number [149]. Some of these show myopathy, together with other organ manifestations.

In fresh, permeabilized muscle, respirometry can be performed using a Clark-type electrode. By using different inhibitors of the respiratory chain-enzymes and uncouplers, the rate-limiting step of respiration can be pinpointed [150, $151]$.

Using ${ }^{31} \mathrm{P}$ MRS, reduction of the ratio of phosphocreatine/inorganic phosphate can be detected, while ATP-levels are normal, phosphocreatine depletion during exercise is larger than normal, and the recovery rate of phosphocreatine after exercise is typically reduced [27].

Mutation analysis may be performed. Both mutations of the nuclear gene and the mitochondrial gene have been reported (see above).

Treatment. Management and support in a multidisciplinary team should be offered to patients and their families. Symptomatic treatment is important in order to prevent or improve morbidity. Seizure-control by antiepileptic drugs (valproic acid should be generally avoided, phenobarbital should be avoided in complex I deficiency) and control of diabetes by insulin are only few examples. A positive energetic balance is desirable; "fast" carbohydrates should be avoided as this may lead to excessive lactic acidosis.

Many single substances have been tried with the rationale of bypassing defective pathways, increasing residual enzyme activities, or having an antioxidant function. For example, succinate has been used to bypass deficient complex I activity, vitamin $\mathrm{C}$ and vitamin $\mathrm{K}$ may bypass deficient complex III, while vitamin E, alphalipoic acid, and coenzyme Q10 have been advocated as antioxidants. However, a recent Cochrane review has stated that none of these as well as other forms of cofactor therapy have been shown to be of any benefit. Only coenzyme Q10 had a positive effect in patients presumably suffering from a disorder in coenzyme Q10 metabolism. Relatively high doses of $2.3 \mathrm{mg} / \mathrm{kg}$ per day are required.

Ketogenic diet has a remarkable effect on CNS-symptoms in PDH-deficiency but has also been tried in complex I deficiency.

Certain drugs inhibiting mitochondrial protein synthesis, like tetracyclines and chloramphenicol, should be avoided.

\section{Miscellaneous}

Some disorders in muscle energy metabolism are very rare and/or have mild symptoms. These are dealt with in this section.

Recently, sudden cardiac death has been reported in a child with muscle-specific glycogen synthase deficiency [152]. In horses, but so far not in humans gain of function mutations of glycogen synthase leading to myopathy has been found [153].
Reduced activity of the phosphorylase kinase, which is needed for glycogen breakdown, has been reported to lead to mild myopathy [154, 155].

Inhibition of the glycolytic pathway at the level of triosephosphate isomerase [156], phosphoglycerate kinase [157], phosphoglycerate mutase [158], and enolase [159] has been described to lead to myopathy with exercise-induced muscle cramps and myoglobinuria.

\section{Special Problems}

Malignant hyperthermia is due to mutations in the ryanodine receptor leading to defects in calcium homeostasis triggered by certain anaesthetic compounds [160-162]. Some neuromuscular diseases are known to be associated with an increased risk of malignant hyperthermia if triggering substances are used for anaesthesia.

Testing for malignant hyperthermia may be performed by the caffeine halothane contracture test or by looking for mutations of the ryanodine receptor gene. As the gene is large and many different mutations are found, it is often safer, faster, and less expensive to perform the caffeine halothane contracture test. However, this involves doing a muscle biopsy. It is often more practical to simply avoid anaesthetic compounds that trigger malignant hyperthermia, rather than doing the contracture test.

An increased risk for malignant hyperthermia has been discussed in several disorders of energy metabolism like CPT2 deficiency [163], respiratory chain defects [164, 165], and McArdle disease [166]. Depolarizing drugs like succinylcholine for muscle relaxation may trigger malignant hyperthermia. Though no firm correlation between inborn errors of energy metabolism and malignant hyperthermia has been established, it is probably wise to avoid triggering substances in these patients.

Furthermore, inhalative anaesthetic agents have been reported to interfere with $\mathrm{NADH}$-linked respiratory chain function [167]. Therefore, these compounds should be avoided in patients with respiratory chain defects. Inhibition of the respiratory chain could lead to free radical generation which may mediate lipid peroxidation in cellular membranes, thus inducing calcium efflux from cell organelles which results in malignant hyperthermia [168].

Psychosocial problems may be another feature in myopathies due to defects in energy metabolism, as more and more patients with inborn errors of metabolism reach adulthood. Inherited neuromuscular disorders affect about one in 3,500 children, with Duchenne muscular dystrophy being the most common disease. To our knowledge, there are no studies on quality of life, and psychosocial problems in patients with myopathies based on compromised energy metabolism. However, studies on the special needs, quality of life and psychosocial problems in boys/men with Duchenne muscular dystrophy have been published [169, 170]. Obviously, inborn errors of energy metabolism are different from Duchenne muscular dystrophy where the physical handicap is enormous. However, it has been shown that quality of life is not correlated with physical handicap in Duchenne muscular dystrophy. Therefore, problems may also be expected in 
Table 1

\begin{tabular}{|c|c|c|}
\hline Disease & Enzyme/Transporter Defect & Main clinical features \\
\hline GSD III (Cori-Forbes) & $\begin{array}{l}\text { Amylo-1,6 glucosidase } \\
\text { (debranching enzyme) }\end{array}$ & $\begin{array}{l}\text { (distal) muscle weakness, cardiomyopathy, hepatomegaly, hypoglycaemia, } \\
\text { growth retardation; } \\
\text { osteoporosis, liver cirrhosis, hepatocellular carcinoma (later phase) }\end{array}$ \\
\hline GSD V (Mc Ardle) & Muscle phosphorylase & Muscle pain (exercise-induced), myoglobinuria \\
\hline GSD VII (Tarui) & $\begin{array}{l}\text { Muscle } \\
\text { phosphofructokinase }\end{array}$ & Muscle pain (+ vomiting, nausea), anaemia \\
\hline CPT2-deficiency & $\begin{array}{l}\text { Carnitine palmitoyl } \\
\text { transferase } 2\end{array}$ & Muscle weakness, cardiomyopathy, hepatomegaly, hypoglycaemia, seizures \\
\hline $\begin{array}{l}\text { MTP (Mitochondrial } \\
\text { trifunctional protein) }\end{array}$ & $\begin{array}{l}\text { LCHAD, thiolase, } \\
\text { enoyl-CoA hydratase }\end{array}$ & $\begin{array}{l}\text { Sudden death, "Reye-like" syndrome, respiratory dysfunction, capilliary } \\
\text { leak syndrome, myopathy, myoglobinuria, cardiomyopathy, peripheral } \\
\text { neuropathy, retinopathy }\end{array}$ \\
\hline VLCAD-deficiency & VLCAD & $\begin{array}{l}\text { Cardiomyopathy, muscle weakness, myoglobinuria, hypoglycaemia, liver } \\
\text { failure }\end{array}$ \\
\hline $\begin{array}{l}\text { MAD-deficiency (Glutaric } \\
\text { aciduria type II) }\end{array}$ & $\begin{array}{l}\text { Electron transfer } \\
\text { flavoprotein, electron } \\
\text { transfer flavoprotein } \\
\text { dehydrogenase }\end{array}$ & $\begin{array}{l}\text { Muscle weakness, cardiomyopathy, hypoglycaemia, respiratory } \\
\text { dysfunction, encephalopathy }\end{array}$ \\
\hline CT-deficiency & Carnitine transporter & $\begin{array}{l}\text { SIDS, "Reye-like" syndrome, encephalopathy, muscle hypotonia, myalgia } \\
\text { (later), psychomotor delay, dilatative cardiomyopathy. } \\
\text { arrythmias, ventricular fibrillations }\end{array}$ \\
\hline Respiratory chain defects & $\begin{array}{l}\text { Respiratory chain } \\
\text { complexes I-V (single or } \\
\text { combined) }\end{array}$ & $\begin{array}{l}\text { Multisystem-disease (e.g., myopathy, cardiomyopathy, hepatopathy, } \\
\text { epilepsy, developmental delay, mental retardation, etc.) }\end{array}$ \\
\hline
\end{tabular}

metabolic myopathies. Further studies are required but may be difficult due to the rarity and high clinical variability of metabolic myopathies.

\section{Summary}

Fatty acids are the main fuel for energy metabolism in heart and skeletal muscle. In the initial anaerobic phase of skeletal muscle contraction, muscular glycogen is used as a substrate.

There are many inborn errors of energy metabolism leading to myopathies which have to be taken into account in the diagnostic work-up. These concern fatty acid oxidation, glycogen metabolism, as well as the "final common pathway", the mitochondrial respiratory chain. For most of the different metabolic disorders, specific therapeutic options exist. Therefore, a metabolic work-up is mandatory in myopathies (skeletal myopathy as well as cardiomyopathy) of unknown origin. An overview, including the most common disorders, is given in Table 1.

\section{References}

[1] A. J. Wagenmakers, "Muscle amino acid metabolism at rest and during exercise: role in human physiology and metabolism," Exercise and Sport Sciences Reviews, vol. 26, pp. 287-314, 1998.

[2] E. Blomstrand and B. Essén-Gustavsson, "Changes in amino acid concentration in plasma and type I and type II fibres during resistance exercise and recovery in human subjects," Amino Acids, vol. 37, no. 4, pp. 629-636, 2008.
[3] J. D. McGarry and N. F. Brown, "The mitochondrial carnitine palmitoyltransferase system. From concept to molecular analysis," European Journal of Biochemistry, vol. 244, no. 1, pp. 1-14, 1997.

[4] A. E. Jeukendrup, W. H. Saris, and A. J. Wagenmakers, "Fat metabolism during exercise: a review-part II: regulation of metabolism and the effects of training," International Journal of Sports Medicine, vol. 19, no. 5, pp. 293-302, 1998.

[5] R. J. A. Wanders, P. Vreken, M. E. J. Den Boer, F. A. Wijburg, A. H. Van Gennip, and L. IJlst, "Disorders of mitochondrial fatty acyl-CoA $\beta$-oxidation," Journal of Inherited Metabolic Disease, vol. 22, no. 4, pp. 442-487, 1999.

[6] P. Mitchell, "Chemiosmotic coupling in energy transduction: a logical development of biochemical knowledge," Journal of Bioenergetics, vol. 3, no. 1-2, pp. 5-24, 1972.

[7] A. M. Das and D. A. Harris, "Intracellular calcium as a regulator of the mitochondrial ATP synthase in cultured cardiomyocytes," Biochemical Society Transactions, vol. 18, no. 4, pp. 554-555, 1990.

[8] A. M. Das and D. A. Harris, "Regulation of the mitochondrial ATP synthase in intact rat cardiomyocytes," Biochemical Journal, vol. 266, no. 2, pp. 355-361, 1990.

[9] A. M. Das, "Regulation of the mitochondrial ATP-synthase in health and disease," Molecular Genetics and Metabolism, vol. 79, no. 2, pp. 71-82, 2003.

[10] D. A. Harris and A. M. Das, "Control of mitochondrial ATP synthesis in the heart," Biochemical Journal, vol. 280, part 3, pp. 561-573, 1991.

[11] A. M. Das, "Regulation of mitochondrial ATP synthase activity in human myocardium," Clinical Science, vol. 94, no. 5, pp. 499-504, 1998. 
[12] E. W. Yamada and N. J. Huzel, "The calcium-binding ATPase inhibitor protein from bovine heart mitochondria. Purification and properties," Journal of Biological Chemistry, vol. 263, no. 23, pp. 11498-11503, 1988.

[13] A. M. Das and D. A. Harris, "Control of mitochondrial ATP synthase in rat cardiomyocytes: effects of thyroid hormone," Biochimica et Biophysica Acta, vol. 1096, no. 4, pp. 284-290, 1991.

[14] A. M. Das and D. A. Harris, "Mitochondrial ATP synthase regulation in heart: defects in hypertension are restored after treatment with captopril," Cardioscience, vol. 3, no. 4, pp. 227-232, 1992.

[15] A. M. Das and D. A. Harris, "Regulation of the mitochondrial ATP synthase is defective in rat heart during alcohol-induced cardiomyopathy," Biochimica et Biophysica Acta, vol. 1181, no. 3, pp. 295-299, 1993.

[16] A. M. Das and D. A. Harris, "Defects in regulation of mitochondrial ATP synthase in cardiomyocytes from spontaneously hypertensive rats," American Journal of Physiology, vol. 259, no. 4, part 2, pp. H1264-H1269, 1990.

[17] R. M. Denton, G. A. Rutter, P. J. Midgley, and J. G. McCormack, "Effects of $\mathrm{Ca}^{2+}$ on the activities of the calciumsensitive dehydrogenases within the mitochondria of mammalian tissues," Journal of Cardiovascular Pharmacology, vol. 12, no. 5, pp. S69-S72, 1988.

[18] J. G. McCormack and R. M. Denton, "The effects of calcium ions and adenine nucleotides on the activity of pig heart 2oxoglutarate dehydrogenase complex," Biochemical Journal, vol. 180, no. 3, pp. 533-544, 1979.

[19] R. M. Denton, "Regulation of mitochondrial dehydrogenases by calcium ions," Biochimica et Biophysica Acta, vol. 1787, no. 11, pp. 1309-1316, 2009.

[20] D. Constantin-Teodosiu, N. S. Peirce, J. Fox, and P. L. Greenhaff, "Muscle pyruvate availability can limit the flux, but not activation, of the pyruvate dehydrogenase complex during submaximal exercise in humans," The Journal of Physiology, vol. 561, no. 2, pp. 647-655, 2004.

[21] G. C. Brown, "Regulation of mitochondrial respiration by nitric oxide inhibition of cytochrome $c$ oxidase," Biochimica et Biophysica Acta, vol. 1504, no. 1, pp. 46-57, 2001.

[22] G. C. Brown, "Nitric oxide and mitochondria," Frontiers in Bioscience, vol. 12, no. 3, pp. 1024-1033, 2007.

[23] L. B. Valdez and A. Boveris, "Mitochondrial nitric oxide synthase, a voltage-dependent enzyme, is responsible for nitric oxide diffusion to cytosol," Frontiers in Bioscience, vol. 12, no. 4, pp. 1210-1219, 2007.

[24] L. B. Valdez, T. Zaobornyj, and A. Boveris, "Mitochondrial metabolic states and membrane potential modulate mtNOS activity," Biochimica et Biophysica Acta, vol. 1757, no. 3, pp. 166-172, 2006.

[25] N. Gordon, "Glycogenosis type V or McArdle's disease," Developmental Medicine and Child Neurology, vol. 45, no. 9, pp. 640-644, 2003.

[26] F. Hanisch, K. Eger, S. Bork, H. Lehnich, M. Deschauer, and S. T. Zierz, "Lactate production upon short-term nonischemic forearm exercise in mitochondrial disorders and other myopathies," Journal of Neurology, vol. 253, no. 6, pp. 735-740, 2006.

[27] D. Bendahan, J. P. Mattéi, G. Kozak-Ribbens, and P. J. Cozzone, "Non invasive investigation of muscle diseases using ${ }^{31} \mathrm{P}$ magnetic resonance spectroscopy: potential in clinical applications," Revue Neurologique, vol. 158, no. 5, part 1, pp. 527-540, 2002.
[28] B. Barbiroli, R. Medori, H. J. Tritschler, et al., "Lipoic (thioctic) acid increases brain energy availability and skeletal muscle performance as shown by in vivo ${ }^{31} \mathrm{P}-\mathrm{MRS}$ in a patient with mitochondrial cytopathy," Journal of Neurology, vol. 242, no. 7, pp. 472-477, 1995.

[29] G. M. Fabrizi, R. Lodi, M. D’Ettorre, et al., “Autosomal dominant limb girdle myopathy with ragged-red fibers and cardiomyopathy a pedigree study by in vivo ${ }^{31} \mathrm{P}-\mathrm{MR}$ spectroscopy indicating a multisystem mitochondrial defect," Journal of the Neurological Sciences, vol. 137, no. 1, pp. 20-27, 1996.

[30] D. E. Befroy, K. Falk Petersen, D. L. Rothman, and G. I. Shulman, "Chapter 21 assessment of in vivo mitochondrial metabolism by magnetic resonance spectroscopy," Methods in Enzymology, vol. 457, pp. 373-393, 2009.

[31] M. Tashiro, T. Fujimoto, M. Itoh, et al., " ${ }^{18}$ F-FDG PET imaging of muscle activity in runners," Journal of Nuclear Medicine, vol. 40, no. 1, pp. 70-76, 1999.

[32] R. Santer, M. Kinner, U. Steuerwald, et al., "Molecular genetic basis and prevalence of glycogen storage disease type IIIA in the Faroe Islands," European Journal of Human Genetics, vol. 9, no. 5, pp. 388-391, 2001.

[33] J. H. Ding, T. De Barsy, B. I. Brown, R. A. Coleman, and Y. T. Chen, "Immunoblot analyses of glycogen debranching enzyme in different subtypes of glycogen storage disease type III," The Journal of Pediatrics, vol. 116, no. 1, pp. 95-100, 1990.

[34] R. A. Coleman, H. S. Winter, B. Wolf, J. M. Gilchrist, and Y. T. Chen, "Glycogen storage disease type III (glycogen debranching enzyme deficiency): correlation of biochemical defects with myopathy and cardiomyopathy," Annals of Internal Medicine, vol. 116, no. 11, pp. 896-900, 1992.

[35] Y. Endo, A. Horinishi, M. Vorgerd, et al., "Molecular analysis of the AGL gene: heterogeneity of mutations in patients with glycogen storage disease type III from Germany, Canada, Afghanistan, Iran, and Turkey," Journal of Human Genetics, vol. 51, no. 11, pp. 958-963, 2006.

[36] S. Lucchiari, D. Santoro, S. Pagliarani, and G. P. Comi, "Clinical, biochemical and genetic features of glycogen debranching enzyme deficiency," Acta Myologica, vol. 26, no. 1, pp. 72-74, 2007.

[37] B. Schoser, D. Gläser, and J. Müller-Höcker, "Clinicopathological analysis of the homozygous p.W1327X AGL mutation in glycogen storage disease type 3," American Journal of Medical Genetics, Part A, vol. 146, no. 22, pp. 2911-2915, 2008.

[38] E. Demo, D. Frush, M. Gottfried, et al., "Glycogen storage disease type III-hepatocellular carcinoma a long-term complication?" Journal of Hepatology, vol. 46, no. 3, pp. 492-498, 2007.

[39] S. Kiechl, J. Willeit, W. Vogel, U. Kohlendorfer, and W. Poewe, "Reversible severe myopathy of respiratory muscles due to adult-onset type III glycogenosis," Neuromuscular Disorders, vol. 9, no. 6-7, pp. 408-410, 1999.

[40] S. Kiechl, U. Kohlendorfer, C. Thaler, et al., "Different clinical aspects of debrancher deficiency myopathy," Journal of Neurology Neurosurgery and Psychiatry, vol. 67, no. 3, pp. 364-368, 1999.

[41] M. A. Kotb, H. K. Abdullah, and A. Kotb, "Liver glycogenoses: are they a possible cause of polyneuropathy? A cross-sectional study," Journal of Tropical Pediatrics, vol. 50, no. 4, pp. 196202, 2004. 
[42] H. R. Mundy, J. E. Williams, P. J. Lee, and M. S. Fewtrell, "Reduction in bone mineral density in glycogenosis type III may be due to a mixed muscle and bone deficit," Journal of Inherited Metabolic Disease, vol. 31, no. 3, pp. 418-423, 2008.

[43] H. Akazawa, T. Kuroda, S. Kim, H. Mito, T. Kojo, and K. Shimada, "Specific heart muscle disease associated with glycogen storage disease type III: clinical similarity to the dilated phase of hypertrophic cardiomyopathy," European Heart Journal, vol. 18, no. 3, pp. 532-533, 1997.

[44] H. Tada, T. Kurita, T. Ohe, et al., "Glycogen storage disease type III associated with ventricular tachycardia," American Heart Journal, vol. 130, no. 4, pp. 911-912, 1995.

[45] P. J. Lee, J. E. Deanfield, M. Burch, K. Baig, W. J. McKenna, and J. V. Leonard, "Comparison of the functional significance of left ventricular hypertrophy in hypertrophic cardiomyopathy and glycogenosis type III," The American Journal of Cardiology, vol. 79, no. 6, pp. 834-838, 1997.

[46] J. J. Shen and Y. T. Chen, "Molecular characterization of glycogen storage disease type III," Current Molecular Medicine, vol. 2, no. 2, pp. 167-175, 2002.

[47] Y. Aoyama, I. Ozer, M. Demirkol, et al., "Molecular features of 23 patients with glycogen storage disease type III in Turkey: a novel mutation p.R1147G associated with isolated glucosidase deficiency, along with 9 AGL mutations," Journal of Human Genetics, vol. 54, no. 11, pp. 681-686, 2009.

[48] J. Seigel, D. A. Weinstein, R. Hillman, B. Colbert, B. Matthews, and B. Bachrach, "Glycogen storage disease type IIIa presenting as non-ketotic hypoglycemia: use of a newly approved commercially available mutation analysis to non-invasively confirm the diagnosis," Journal of Pediatric Endocrinology and Metabolism, vol. 21, no. 6, pp. 587-590, 2008.

[49] A. I. Dagli, R. T. Zori, H. McCune, T. Ivsic, M. K. Maisenbacher, and D. A. Weinstein, "Reversal of glycogen storage disease type IIIa-related cardiomyopathy with modification of diet," Journal of Inherited Metabolic Disease, pp. 1-4, 2009.

[50] C. Bartram, R. H. T. Edwards, J. Clague, and R. J. Beynon, "McArdle's disease: a nonsense mutation in exon 1 of the muscle glycogen phosphorylase gene explains some but not all cases," Human Molecular Genetics, vol. 2, no. 8, pp. 12911293, 1993.

[51] C. Kubisch, E. M. Wicklein, and T. J. Jentsch, "Molecular diagnosis of McArdle disease: revised genomic structure of the myophosphorylase gene and identification of a novel mutation," Human Mutation, vol. 12, no. 1, pp. 27-32, 1998.

[52] A. L. Andreu, G. Nogales-Gadea, D. Cassandrini, J. Arenas, and C. Bruno, "McArdle disease: molecular genetic update," Acta Myologica, vol. 26, no. 1, pp. 53-57, 2007.

[53] F. Gómez-Gallego, C. Santiago, M. Morán, et al., “The I allele of the ACE gene is associated with improved exercise capacity in women with McArdle disease," British Journal of Sports Medicine, vol. 42, no. 2, pp. 134-140, 2008.

[54] A. Lucia, F. Gómez-Gallego, C. Santiago, et al., "The 577X allele of the ACTN3 gene is associated with improved exercise capacity in women with McArdle's disease," Neuromuscular Disorders, vol. 17, no. 8, pp. 603-610, 2007.

[55] A. Martinuzzi, A. Liava, E. Trevisi, L. Antoniazzi, and M. Frare, "Chronic therapy for McArdle disease: the randomized trial with ACE inhibitor," Acta Myologica, vol. 26, no. 1, pp. 64-66, 2007.

[56] B. Mcardle, "Myopathy due to a defect in muscle glycogen breakdown," Clinical Science, vol. 10, pp. 13-33, 1951.
[57] A. A. Nadaj-Pakleza, C. M. Vincitorio, P. Laforêt, et al., "Permanent muscle weakness in McArdle disease," Muscle and Nerve, vol. 40, no. 3, pp. 350-357, 2009.

[58] D. Bendahan, S. Confort-Gouny, G. Kozak-Ribbens, and P. J. Cozzone, "31-P NMR characterization of the metabolic anomalies associated with the lack of glycogen phosphorylase activity in human forearm muscle," Biochemical and Biophysical Research Communications, vol. 185, no. 1, pp. 16-21, 1992.

[59] B. D. Ross, G. K. Radda, D. G. Gadian, G. Rocker, M. Esiri, and J. Falconer-Smith, "Examination of a case of suspected McArdle's syndrome by ${ }^{31} \mathrm{P}$ nuclear magnetic resonance," New England Journal of Medicine, vol. 304, no. 22, pp. 13381342, 1981.

[60] J. L. Fleckenstein, R. G. Haller, S. F. Lewis, et al., "Absence of exercise-induced MRI enhancement of skeletal muscle in McArdle's disease," Journal of Applied Physiology, vol. 71, no. 3, pp. 961-969, 1991.

[61] R. Quinlivan, R. J. Beynon, and A. Martinuzzi, "Pharmacological and nutritional treatment for McArdle disease (Glycogen Storage Disease type V)," Cochrane Database of Systematic Reviews (Online), no. 2, Article ID CD003458, 2008.

[62] M. Vorgerd, T. Grehl, M. Jäger, et al., "Creatine therapy in myophosphorylase deficiency (McArdle disease): a placebocontrolled crossover trial," Archives of Neurology, vol. 57, no. 7, pp. 956-963, 2000.

[63] M. Vorgerd, J. Zange, R. Kley, et al., "Effect of high-dose creatine therapy on symptoms of exercise intolerance in McArdle disease: double-blind, placebo-controlled crossover study," Archives of Neurology, vol. 59, no. 1, pp. 97-101, 2002.

[64] A. Toscano and O. Musumeci, "Tarui disease and distal glycogenoses: clinical and genetic update," Acta Myologica, vol. 26, no. 2, pp. 105-107, 2007.

[65] S. Vora, "Isozymes of phosphofructokinase," Isozymes, vol. 6, pp. 119-167, 1982.

[66] S. Tarui, G. Okuno, Y. Ikura, T. Tanaka, M. Suda, and M. Nishikawa, "Phosphofructokinase deficiency in skeletal muscle. A new type of glycogenosis," Biochemical and Biophysical Research Communications, vol. 19, no. 4, pp. 517-523, 1965.

[67] D. Duboc, P. Jehenson, and S. T. Dinh, "Phosphorus NMR spectroscopy study of muscular enzyme deficiencies involving glycogenolysis and glycolysis," Neurology, vol. 37, no. 4, pp. 663-671, 1987.

[68] Z. Argov, N. De Stefano, and D. L. Arnold, "Muscle highenergy phosphates in central nervous system disorders. The phosphorus MRS experience," The Italian Journal of Neurological Sciences, vol. 18, no. 6, pp. 353-357, 1997.

[69] P. Rinaldo, D. Matern, and M. J. Bennett, "Fatty acid oxidation disorders," Annual Review of Physiology, vol. 64, pp. 477-502, 2002.

[70] S. DiMauro and P. M. M. DiMauro, "Muscle carnitine palmityltransferase deficiency and myoglobinuria," Science, vol. 182, no. 4115, pp. 929-931, 1973.

[71] S. Illsinger, T. Lücke, M. Peter, et al., "Carnitinepalmitoyltransferase 2 deficiency: novel mutations and relevance of newborn screening," American Journal of Medical Genetics, Part A, vol. 146, no. 22, pp. 2925-2928, 2008.

[72] S. Corti, A. Bordoni, D. Ronchi, et al., "Clinical features and new molecular findings in Carnitine Palmitoyltransferase II (CPT II) deficiency," Journal of the Neurological Sciences, vol. 266, no. 1-2, pp. 97-103, 2008. 
[73] A. M. Das, R. Fingerhut, R. J. A. Wanders, and K. Ullrich, "Secondary respiratory chain defect in a boy with long-chain 3- hydroxyacyl-CoA dehydrogenase deficiency: possible diagnostic pitfalls," European Journal of Pediatrics, vol. 159, no. 4, pp. 243-246, 2000.

[74] U. Spiekerkoetter, M. Lindner, R. Santer, et al., "Treatment recommendations in long-chain fatty acid oxidation defects: consensus from a workshop," Journal of Inherited Metabolic Disease, vol. 32, no. 4, pp. 498-505, 2009.

[75] J. O. Solis and R. H. Singh, "Management of fatty acid oxidation disorders: a survey of current treatment strategies," Journal of the American Dietetic Association, vol. 102, no. 12, pp. 1800-1803, 2002.

[76] U. Spiekerkoetter, M. Lindner, R. Santer, et al., "Management and outcome in 75 individuals with long-chain fatty acid oxidation defects: results from a workshop," Journal of Inherited Metabolic Disease, vol. 32, no. 4, pp. 488-497, 2009.

[77] M. B. Gillingham, B. Scott, D. Elliott, and C. O. Harding, "Metabolic control during exercise with and without medium-chain triglycerides (MCT) in children with longchain 3-hydroxy acyl-CoA dehydrogenase (LCHAD) or trifunctional protein (TFP) deficiency," Molecular Genetics and Metabolism, vol. 89, no. 1-2, pp. 58-63, 2006.

[78] M. B. Gillingham, J. Q. Purnell, J. Jordan, D. Stadler, A. M. Haqq, and C. O. Harding, "Effects of higher dietary protein intake on energy balance and metabolic control in children with long-chain 3-hydroxy acyl-CoA dehydrogenase (LCHAD) or trifunctional protein (TFP) deficiency," Molecular Genetics and Metabolism, vol. 90, no. 1, pp. 64-69, 2007.

[79] C. R. Roe, L. Sweetman, D. S. Roe, F. David, and H. Brunengraber, "Treatment of cardiomyopathy and rhabdomyolysis in long-chain fat oxidation disorders using an anaplerotic odd-chain triglyceride," Journal of Clinical Investigation, vol. 110, no. 2, pp. 259-269, 2002.

[80] S. Gobin-Limballe, F. Djouadi, F. Aubey, et al., "Genetic basis for correction of very-long-chain acyl-coenzyme A dehydrogenase deficiency by bezafibrate in patient fibroblasts: toward a genotype-based therapy," American Journal of Human Genetics, vol. 81, no. 6, pp. 1133-1143, 2007.

[81] A. Minnich, N. Tian, L. Byan, and G. Bilder, "A potent PPAR $\alpha$ agonist stimulates mitochondrial fatty acid $\beta$-oxidation in liver and skeletal muscle," American Journal of Physiology, vol. 280, no. 2, pp. E270-E279, 2001.

[82] F. Djouadi, F. Aubey, D. Schlemmer, et al., "Potential of fibrates in the treatment of fatty acid oxidation disorders: revival of classical drugs?" Journal of Inherited Metabolic Disease, vol. 29, no. 2-3, pp. 341-342, 2006.

[83] J. L. K. Van Hove, S. Grünewald, J. Jaeken, et al., "D,L-3hydroxybutyrate treatment of multiple acyl-CoA dehydrogenase deficiency (MADD)," Lancet, vol. 361, no. 9367, pp. 1433-1435, 2003.

[84] C. Gellera, E. Verderio, G. Floridia, et al., "Assignment of the human carnitine palmitoyltransferase II gene (CPT1) to chromosome 1p32," Genomics, vol. 24, no. 1, pp. 195-197, 1994.

[85] M. Deschauer, T. Wieser, and S. Zierz, "Muscle carnitine palmitoyltransferase II deficiency: clinical and molecular genetic features and diagnostic aspects," Archives of Neurology, vol. 62, no. 1, pp. 37-41, 2005.

[86] S. E. Olpin, A. Afifi, S. Clark, et al., "Mutation and biochemical analysis in carnitine palmitoyltransferase type II (CPT II) deficiency," Journal of Inherited Metabolic Disease, vol. 26, no. 6, pp. 543-557, 2003.
[87] G. Hug, K. E. Bove, and S. Soukup, "Lethal neonatal multiorgan deficiency of carnitine palmitoyltransferase II," New England Journal of Medicine, vol. 325, no. 26, pp. 1862$1864,1991$.

[88] F. Taroni and G. Uziel, "Fatty acid mitochondrial $\beta$-oxidation and hypoglycaemia in children," Current Opinion in Neurology, vol. 9, no. 6, pp. 477-485, 1996.

[89] F. Demaugre, J. P. Bonnefont, M. Colonna, C. Cepanec, J. P. Leroux, and J. M. Saudubray, "Infantile form of carnitine palmitoyltransferase II deficiency with hepatomuscular symptoms and sudden death. Physiopathological approach to carnitine palmitoyltransferase II deficiencies," Journal of Clinical Investigation, vol. 87, no. 3, pp. 859-864, 1991.

[90] L. Thuillier, H. Rostane, V. Droin, et al., "Correlation between genotype, metabolic data, and clinical presentation in carnitine palmitoyltransferase 2 (CPT2) deficiency," Human Mutation, vol. 21, no. 5, pp. 493-501, 2003.

[91] G. D. Vladutiu, M. J. Bennett, D. Smail, L. J. Wong, R. T. Taggart, and H. B. Lindsley, "A variable myopathy associated with heterozygosity for the R503C mutation in the carnitine palmitoyltransferase II gene," Molecular Genetics and Metabolism, vol. 70, no. 2, pp. 134-141, 2000.

[92] L. Thuillier, C. Sevin, F. Demaugre, et al., "Genotype/phenotype correlation in carnitine palmitoyl transferase II deficiency: lessons from a compound heterozygous patient," Neuromuscular Disorders, vol. 10, no. 3, pp. 200205, 2000.

[93] P. J. Isackson, M. J. Bennett, and G. D. Vladutiu, "Identification of 16 new disease-causing mutations in the CPT2 gene resulting in carnitine palmitoyltransferase II deficiency," Molecular Genetics and Metabolism, vol. 89, no. 4, pp. 323331, 2006.

[94] P. J. Isackson, M. J. Bennett, U. Lichter-Konecki, et al., "CPT2 gene mutations resulting in lethal neonatal or severe infantile carnitine palmitoyltransferase II deficiency," Molecular Genetics and Metabolism, vol. 94, no. 4, pp. 422-427, 2008.

[95] J. P. Bonnefont, F. Taroni, P. Cavadini, et al., "Molecular analysis of carnitine palmitoyltransferase II deficiency with hepatocardiomuscular expression," American Journal of Human Genetics, vol. 58, no. 5, pp. 971-978, 1996.

[96] U. Spiekerkoetter, Z. Khuchua, Z. Yue, M. J. Bennett, and A. W. Strauss, "General mitochondrial trifunctional protein (TFP) deficiency as a result of either $\alpha$ - or $\beta$-subunit mutations exhibits similar phenotypes because mutations in either subunit alter TFP complex expression and subunit turnover," Pediatric Research, vol. 55, no. 2, pp. 190-196, 2004.

[97] R. J. A. Wanders, L. Ijlst, F. Poggi, et al., "Human trifunctional protein deficiency: a new disorder of mitochondrial fatty acid $\beta$-oxidation," Biochemical and Biophysical Research Communications, vol. 188, no. 3, pp. 1139-1145, 1992.

[98] A. M. Das, S. Illsinger, T. Lücke, et al., "Isolated mitochondrial long-chain ketoacyl-CoA thiolase deficiency resulting from mutations in the HADHB gene," Clinical Chemistry, vol. 52, no. 3, pp. 530-534, 2006.

[99] O. Scheuerman, R. J. A. Wanders, H. R. Waterham, G. Dubnov-Raz, and B.-Z. Garty, "Mitochondrial trifunctional protein deficiency with recurrent rhabdomyolysis," Pediatric Neurology, vol. 40, no. 6, pp. 465-467, 2009.

[100] M. E. J. Den Boer, C. Dionisi-Vici, A. Chakrapani, A. O. J. Van Thuijl, R. J. A. Wanders, and F. A. Wijburg, "Mitochondrial trifunctional protein deficiency: a severe fatty acid oxidation disorder with cardiac and neurologic involvement," The Journal of Pediatrics, vol. 142, no. 6, pp. 684-689, 2003. 
[101] K. Izai, Y. Uchida, T. Orii, S. Yamamoto, and T. Hashimoto, "Novel fatty acid beta-oxidation enzymes in rat liver mitochondria. I. Purification and properties of very-long-chain acyl-coenzyme A dehydrogenase," The Journal of Biological Chemistry, vol. 267, no. 2, pp. 1027-1033, 1992.

[102] B. S. Andresen, S. Olpin, B. J. H. M. Poorthuis, et al., "Clear correlation of genotype with disease phenotype in very-longchain acyl-CoA dehydrogenase deficiency," American Journal of Human Genetics, vol. 64, no. 2, pp. 479-494, 1999.

[103] B. S. Andresen, C. Vianey-Saban, P. Bross, et al., "The mutational spectrum in very long-chain acyl-CoA dehydrogenase deficiency," Journal of Inherited Metabolic Disease, vol. 19, no. 2, pp. 169-172, 1996.

[104] C. Bertrand, C. Largilliere, M. T. Zabot, M. Mathieu, and C. Vianey-Saban, "Very long chain acyl-CoA dehydrogenase deficiency: identification of a new inborn error of mitochondrial fatty acid oxidation in fibroblasts," Biochimica et Biophysica Acta, vol. 1180, no. 3, pp. 327-329, 1993.

[105] C. Vianey-Saban, P. Divry, M. Brivet, et al., "Mitochondrial very-long-chain acyl-coenzyme A dehydrogenase deficiency: clinical characteristics and diagnostic considerations in 30 patients," Clinica Chimica Acta, vol. 269, no. 1, pp. 43-62, 1998.

[106] S. Kluge, P. Kühnelt, A. Block, et al., "A young woman with persistent hypoglycemia, rhabdomyolysis, and coma: recognizing fatty acid oxidation defects in adults," Critical Care Medicine, vol. 31, no. 4, pp. 1273-1276, 2003.

[107] A. Boneh, B. S. Andresen, N. Gregersen, et al., "VLCAD deficiency: pitfalls in newborn screening and confirmation of diagnosis by mutation analysis," Molecular Genetics and Metabolism, vol. 88, no. 2, pp. 166-170, 2006.

[108] J. D. Hoffman, R. D. Steiner, L. Paradise, et al., "Rhabdomyolysis in the military: recognizing late-onset very long-chain acyl Co-A dehydrogenase deficiency," Military Medicine, vol. 171, no. 7, pp. 657-658, 2006.

[109] B. Merinero, S. I. Pascual Pascual, C. Pérez-Cerdá, et al., "Adolescent myopathic presentation in two sisters with very long-chain acyl-CoA dehydrogenase deficiency," Journal of Inherited Metabolic Disease, vol. 22, no. 7, pp. 802-810, 1999.

[110] C. Minetti, B. Garavaglia, M. Bado, et al., "Very-long-chain acyl-coenzyme A dehydrogenase deficiency in a child with recurrent myoglobinuria," Neuromuscular Disorders, vol. 8, no. 1, pp. 3-6, 1998.

[111] I. Ogilvie, M. Pourfarzam, S. Jackson, C. Stockdale, K. Bartlett, and D. M. Turnbull, "Very long-chain acyl coenzyme A dehydrogenase deficiency presenting with exercise-induced myoglobinuria," Neurology, vol. 44, no. 3, part 1, pp. 467473, 1994.

[112] R. Pons, P. Cavadini, S. Baratta, et al., "Clinical and molecular heterogeneity in very-long-chain acyl-coenzyme A dehydrogenase deficiency," Pediatric Neurology, vol. 22, no. 2, pp. 98-105, 2000.

[113] H. R. Scholte, R. N. A. Van Coster, P. C. De Jonge, et al., "Myopathy in very-long-chain acyl-CoA dehydrogenase deficiency: clinical and biochemical differences with the fatal cardiac phenotype," Neuromuscular Disorders, vol. 9, no. 5, pp. 313-319, 1999.

[114] A. H. M. Smelt, B. J. H. M. Poorthuis, W. Onkenhout, et al., "Very long chain acyl-coenzyme A dehydrogenase deficiency with adult onset," Annals of Neurology, vol. 43, no. 4, pp. 540$544,1998$.

[115] N. C. Voermans, B. G. van Engelen, L. A. Kluijtmans, N. M. Stikkelbroeck, and A. R. Hermus, "Rhabdomyolysis caused by an inherited metabolic disease: very long-chain Acyl-CoA dehydrogenase deficiency," American Journal of Medicine, vol. 119, no. 2, pp. 176-179, 2006.

[116] A. Zia, E. H. Kolodny, and G. M. Pastores, "Very long chain acyl-CoA dehydrogenase deficiency in a pair of mildly affected monozygotic twin sister in their late fifties," Journal of Inherited Metabolic Disease, vol. 30, no. 5, p. 817, 2007.

[117] P. Laforêt, C. Acquaviva-Bourdain, O. Rigal, et al., "Diagnostic assessment and long-term follow-up of 13 patients with Very Long-Chain Acyl-Coenzyme A dehydrogenase (VLCAD) deficiency," Neuromuscular Disorders, vol. 19, no. 5, pp. 324-329, 2009.

[118] F. E. Frerman and S. I. Goodman, "Defects of electron transfer flavoprotein and electron transfer flavoproteinubiquinone oxidoreductase: glutaric acidaemia type II," in The Metabolic and Molecular Bases of Inherited Disease, C. R. Scriver, A. L. Beaudet, W. S. Sly, and D. Valle, Eds., pp. 23572365, McGraw-Hill, New York, NY, USA, 8th edition, 2001.

[119] R. K. J. Olsen, M. Pourfarzam, A. A. M. Morris, et al., "Lipid-storage myopathy and respiratory insufficiency due to ETFQO mutations in a patient with late-onset multiple acyl-CoA dehydrogenation deficiency," Journal of Inherited Metabolic Disease, vol. 27, no. 5, pp. 671-678, 2004.

[120] E. Christensen, S. Kolvraa, and N. Gregersen, "Glutaric aciduria type II: evidence for a defect related to the electron transfer flavoprotein or its dehydrogenase," Pediatric Research, vol. 18, no. 7, pp. 663-667, 1984.

[121] N. Gregersen, B. S. Andresen, C. B. Pedersen, R. K. J. Olsen, T. J. Corydon, and P. Bross, "Mitochondrial fatty acid oxidation defects—remaining challenges," Journal of Inherited Metabolic Disease, vol. 31, no. 5, pp. 643-657, 2008.

[122] R. K. J. Olsen, S. E. Olpin, B. S. Andresen, et al., "ETFDH mutations as a major cause of riboflavin-responsive multiple acyl-CoA dehydrogenation deficiency," Brain, vol. 130, no. 8, pp. 2045-2054, 2007.

[123] B. Wen, T. Dai, W. Li, et al., "Riboflavin-responsive lipidstorage myopathy caused by ETFDH gene mutations," Journal of Neurology, Neurosurgery and Psychiatry, vol. 81, no. 2, pp. 231-236, 2010.

[124] J. Bremer, "Carnitine-metabolism and functions," Physiological Reviews, vol. 63, no. 4, pp. 1420-1480, 1983.

[125] I. Tein, "Carnitine transport: pathophysiology and metabolism of known molecular defects," Journal of Inherited Metabolic Disease, vol. 26, no. 2-3, pp. 147-169, 2003.

[126] P. Rinaldo, C. A. Stanley, B. Y. L. Hsu, L. A. Sanchez, and H. J. Stern, "Sudden neonatal death in carnitine transporter deficiency," The Journal of Pediatrics, vol. 131, no. 2, pp. 304305, 1997.

[127] C. A. Stanley, S. DeLeeuw, P. M. Coates, et al., "Chronic cardiomyopathy and weakness or acute coma in children with a defect in carnitine uptake," Annals of Neurology, vol. 30, no. 5, pp. 709-716, 1991.

[128] R. Pons and D. C. De Vivo, "Primary and secondary carnitine deficiency syndromes," Journal of Child Neurology, vol. 10, supplement 2, pp. S8-S24, 1995.

[129] I. Tein, D. C. De Vivo, F. Bierman, et al., "Impaired skin fibroblast carnitine uptake in primary systemic carnitine deficiency manifested by childhood carnitine-responsive cardiomyopathy," Pediatric Research, vol. 28, no. 3, pp. 247255, 1990.

[130] A. M. Lamhonwah, S. E. Olpin, R. J. Pollitt, et al., "Novel OCTN2 mutations: No genotype-phenotype correlations: early carnitine therapy prevents cardiomyopathy," American Journal of Medical Genetics, vol. 111, no. 3, pp. 271-284, 2002. 
[131] R. S. Rijlaarsdam, F. J. van Spronsen, M. T. H. E. BinkBoelkens, et al., "Ventricular fibrillation without overt cardiomyopathy as first presentation of organic cation transporter 2-deficiency in adolescence," Pacing and Clinical Electrophysiology, vol. 27, no. 5, pp. 675-676, 2004.

[132] S. Vielhaber, H. Feistner, J. Weis, et al., "Primary carnitine deficiency: adult onset lipid storage myopathy with a mild clinical course," Journal of Clinical Neuroscience, vol. 11, no. 8, pp. 919-924, 2004.

[133] U. Spiekerkoetter, G. Huener, T. Baykal, et al., "Silent and symptomatic primary carnitine deficiency within the same family due to identical mutations in the organic cation/carnitine transporter OCTN2," Journal of Inherited Metabolic Disease, vol. 26, no. 6, pp. 613-615, 2003.

[134] S. Vijay, A. Patterson, S. Olpin, et al., "Carnitine transporter defect: diagnosis in asymptomatic adult women following analysis of acylcarnitines in their newborn infants," Journal of Inherited Metabolic Disease, vol. 29, no. 5, pp. 627-630, 2006.

[135] Y. Wang, S. H. Korman, J. Ye, et al., "Phenotype and genotype variation in primary carnitine deficiency," Genetics in Medicine, vol. 3, no. 6, pp. 387-392, 2001.

[136] A. M. Das and D. A. Harris, "Control of mitochondrial ATP synthase in heart cells: inactive to active transitions caused by beating or positive inotropic agents," Cardiovascular Research, vol. 24, no. 5, pp. 411-417, 1990.

[137] A. M. Das and D. J. Byrd, "Regulation of the mitochondrial ATP-synthase in skeletal muscle from children-a new diagnostic tool," Journal of Inherited Metabolic Disease, vol. 19, no. 2, pp. 137-139, 1996.

[138] D. G. Nicholls and S. L. Budd, "Mitochondria and neuronal survival," Physiological Reviews, vol. 80, no. 1, pp. 315-360, 2000.

[139] S. Raha and B. H. Robinson, "Mitochondria, oxygen free radicals, and apoptosis," American Journal of Medical Genetics, vol. 106, no. 1, pp. 62-70, 2001.

[140] R. Luft, D. Ikkos, G. Palmieri, L. Ernster, and B. Afzelius, "A case of severe hypermetabolism of nonthyroid origin with a defect in the maintenance of mitochondrial respiratory control: a correlated clinical, biochemical, and morphological study," The Journal of Clinical Investigation, vol. 41, no. 9, pp. 1776-1804, 1962.

[141] L. Ernster and R. Luft, "Further studies on a population of human skeletal muscle mitochondria lacking respiratory control," Experimental Cell Research, vol. 32, no. 1, pp. 2635, 1963.

[142] F. G. Debray, G. A. Mitchell, P. Allard, B. H. Robinson, J. A. Hanley, and M. Lambert, "Diagnostic accuracy of blood lactate-to-pyruvate molar ratio in the differential diagnosis of congenital lactic acidosis," Clinical Chemistry, vol. 53, no. 5, pp. 916-921, 2007.

[143] B. H. Robinson, D. M. Glerum, W. Chow, R. PetrovaBenedict, R. Lightowlers, and R. Capaldi, "The use of skin fibroblast cultures in the detection of respiratory chain defects in patients with lacticacidemia," Pediatric Research, vol. 28, no. 5, pp. 549-555, 1990.

[144] N. Mackay and B. H. Robinson, "Measurement of the ratio of lactate to pyruvate in skin fibroblast cultures," Methods in Cell Biology, vol. 80, pp. 173-178, 2007.

[145] A. M. Das and K. Ullrich, "Dysregulation of the mitochondrial ATP-synthase in respiratory chain defects: first experience," Journal of Inherited Metabolic Disease, vol. 21, no. 3, pp. 220-223, 1998.

[146] A. Abadi, E. I. Glover, R. J. Isfort, et al., "Limb immobilization induces a coordinate down-regulation of mitochondrial and other metabolic pathways in men and women," PLoS One, vol. 4, no. 8, Article ID e6518, 2009.

[147] L.-J. C. Wong, "Comprehensive molecular diagnosis of mitochondrial disorders: qualitative and quantitative approach," Annals of the New York Academy of Sciences, vol. 1011, pp. 246-258, 2004.

[148] L.-J. C. Wong, "Diagnostic challenges of mitochondrial DNA disorders," Mitochondrion, vol. 7, no. 1-2, pp. 45-52, 2007.

[149] C. T. Moraes, S. Shanske, H. J. Tritschler, et al., "mtDNA depletion with variable tissue expression: a novel genetic abnormality in mitochondrial diseases," American Journal of Human Genetics, vol. 48, no. 3, pp. 492-501, 1991.

[150] W. Sperl, D. Skladal, E. Gnaiger, et al., "High resolution respirometry of permeabilized skeletal muscle fibers in the diagnosis of neuromuscular disorders," Molecular and Cellular Biochemistry, vol. 174, no. 1-2, pp. 71-78, 1997.

[151] S. Vielhaber, A. Kudin, R. Schröder, C. E. Elger, and W. S. Kunz, "Muscle fibres: applications for the study of the metabolic consequences of enzyme deficiencies in skeletal muscle," Biochemical Society Transactions, vol. 28, no. 2, pp. 159-164, 2000.

[152] J. M. Cameron, V. Levandovskiy, N. MacKay, et al., "Identification of a novel mutation in GYS1 (muscle-specific glycogen synthase) resulting in sudden cardiac death, that is diagnosable from skin fibroblasts," Molecular Genetics and Metabolism, vol. 98, no. 4, pp. 378-382, 2009.

[153] M. E. McCue, S. J. Valberg, M. B. Miller, et al., "Glycogen synthase (GYS1) mutation causes a novel skeletal muscle glycogenosis," Genomics, vol. 91, no. 5, pp. 458-466, 2008.

[154] W. I. M. Wuyts, E. Reyniers, C. Ceuterick, K. Storm, T. De Barsy, and J. J. Martin, "Myopathy and phosphorylase kinase deficiency caused by a mutation in the PHKA1 gene," American Journal of Medical Genetics, vol. 133, no. 1, pp. 8284, 2005.

[155] M. C. Ørngreen, H. J. Schelhaas, T. D. Jeppesen, et al., "Is muscle glycogenolysis impaired in X-linked phosphorylase $b$ kinase deficiency?" Neurology, vol. 70, no. 20, pp. 1876-1882, 2008.

[156] B. T. Poll-The, J. Aicardi, R. Girot, and R. Rosa, "Neurological findings in triosephosphate isomerase deficiency," Annals of Neurology, vol. 17, no. 5, pp. 439-443, 1985.

[157] R. Spiegel, E. A. Gomez, H. O. Akman, S. Krishna, Y. Horovitz, and S. DiMauro, "Myopathic form of phosphoglycerate kinase (PGK) deficiency: a new case and pathogenic considerations," Neuromuscular Disorders, vol. 19, no. 3, pp. 207-211, 2009.

[158] S. J. Oh, K. S. Park, H. F. Ryan, et al., "Exercise-induced cramp, myoglobinuria, and tubular aggregates in phosphoglycerate mutase deficiency," Muscle and Nerve, vol. 34, no. 5, pp. 572-576, 2006.

[159] G. P. Comi, F. Fortunato, S. Lucchiari, et al., " $\beta$-enolase deficiency, a new metabolic myopathy of distal glycolysis," Annals of Neurology, vol. 50, no. 2, pp. 202-207, 2001.

[160] T. E. Nelson, "Malignant hyperthermia: a pharmacogenetic disease of Ca regulating proteins," Current Molecular Medicine, vol. 2, no. 4, pp. 347-369, 2002.

[161] M. Steinfath, F. Wappler, and J. Scholz, "Malignant hyperthermia. General, clinical and experimental aspects," Der Anaesthesist, vol. 51, no. 4, pp. 328-345, 2002.

[162] H. Rosenberg, M. Davis, D. James, N. Pollock, and K. Stowell, "Malignant hyperthermia," Orphanet Journal of Rare Diseases, vol. 2, article 21, 2007.

[163] G. D. Vladutiu, K. Hogan, I. Saponara, L. Tassini, and J. Conroy, "Carnitine palmitoyl transferase deficiency in 
malignant hyperthermia," Muscle and Nerve, vol. 16, no. 5, pp. 485-491, 1993.

[164] A. K. W. Brownell, "Malignant hyperthermia: relationship to other diseases," British Journal of Anaesthesia, vol. 60, no. 3, pp. 303-308, 1988.

[165] E. W. S. Cheam and L. A. H. Critchley, "Anesthesia for a child with complex I respiratory chain enzyme deficiency," Journal of Clinical Anesthesia, vol. 10, no. 6, pp. 524-527, 1998.

[166] J. Benca and K. Hogan, "Malignant hyperthermia, coexisting disorders, and enzymopathies: risks and management options," Anesthesia and Analgesia, vol. 109, no. 4, pp. 10491053, 2009.

[167] G. M. Hall, S. J. Kirtland, and H. Baum, "The inhibition of mitochondrial respiration by inhalational anaesthetic agents," British Journal of Anaesthesia, vol. 45, no. 10, pp. 1005-1009, 1973.

[168] G. G. Duthie and J. R. Arthur, "Free radicals and calcium homeostasis: relevance to malignant hyperthermia?" Free Radical Biology and Medicine, vol. 14, no. 4, pp. 435-442, 1993.

[169] J. Rahbek, B. Werge, A. Madsen, J. Marquardt, B. F. Steffensen, and J. Jeppesen, "Adult life with Duchenne muscular dystrophy: observations among an emerging and unforeseen patient population," Pediatric Rehabilitation, vol. 8, no. 1, pp. 17-28, 2005.

[170] E.-M. Strehle, "Long-term management of children with neuromuscular disorders," Jornal de Pediatria, vol. 85, no. 5, pp. 379-384, 2009. 

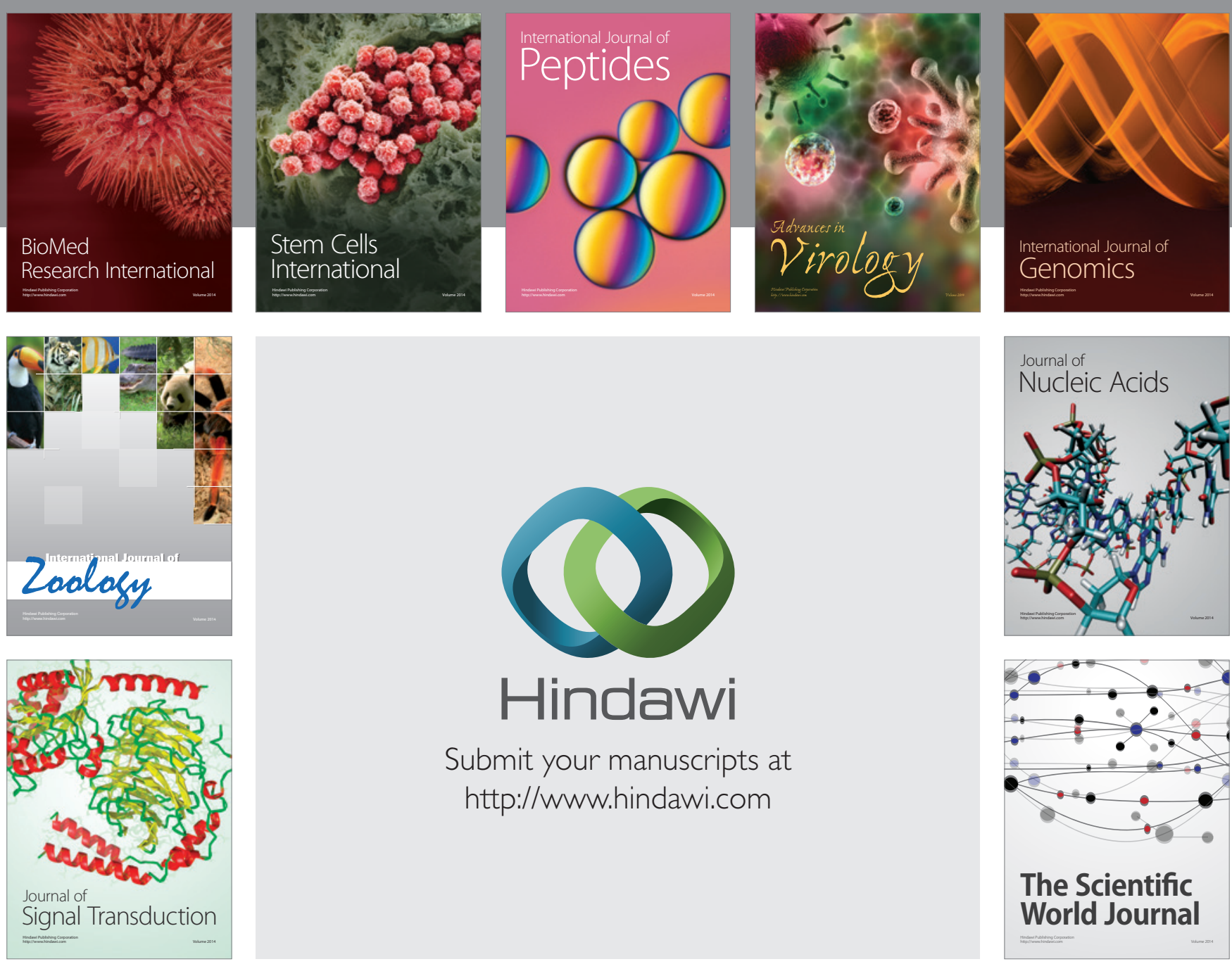

Submit your manuscripts at

http://www.hindawi.com
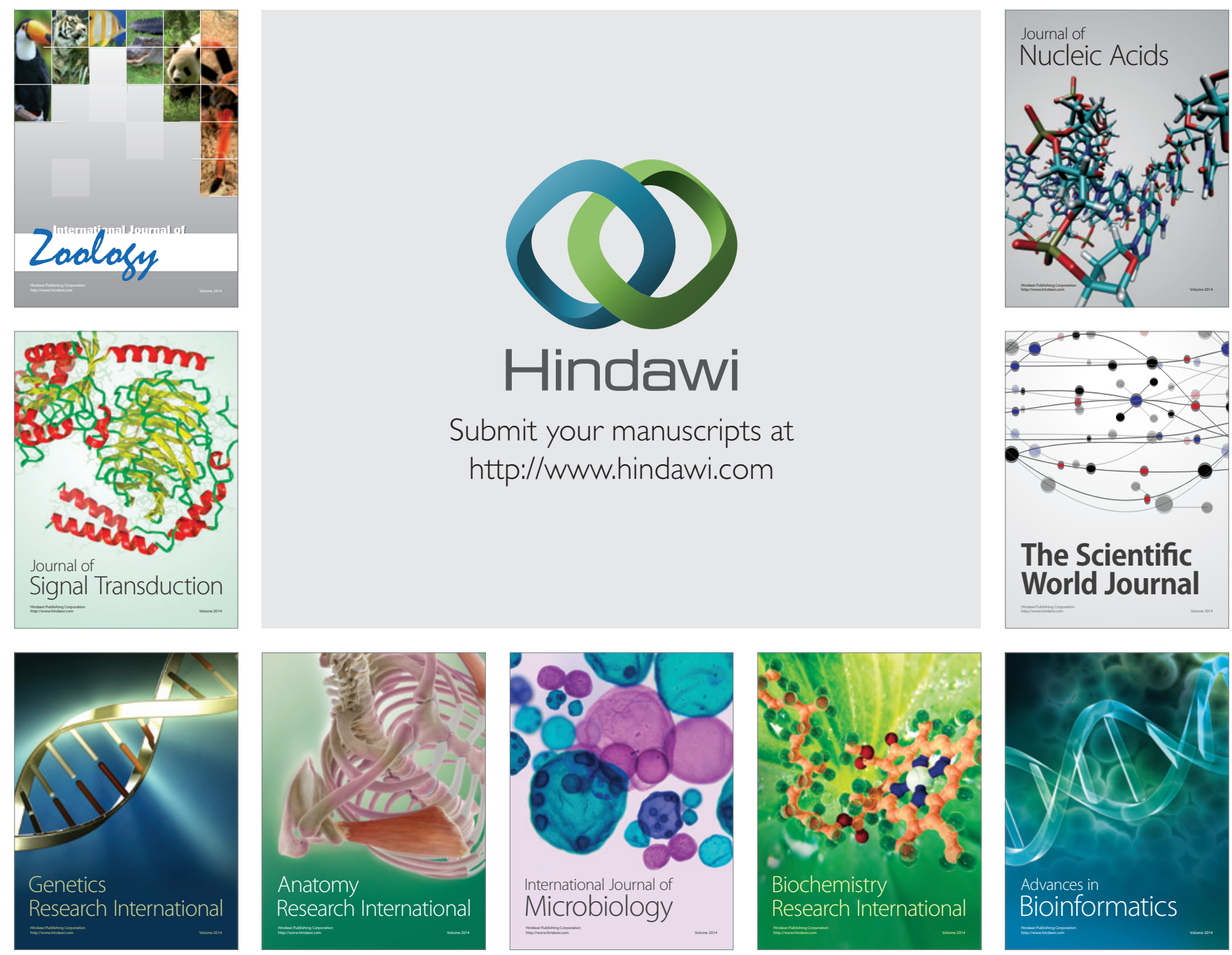

The Scientific World Journal
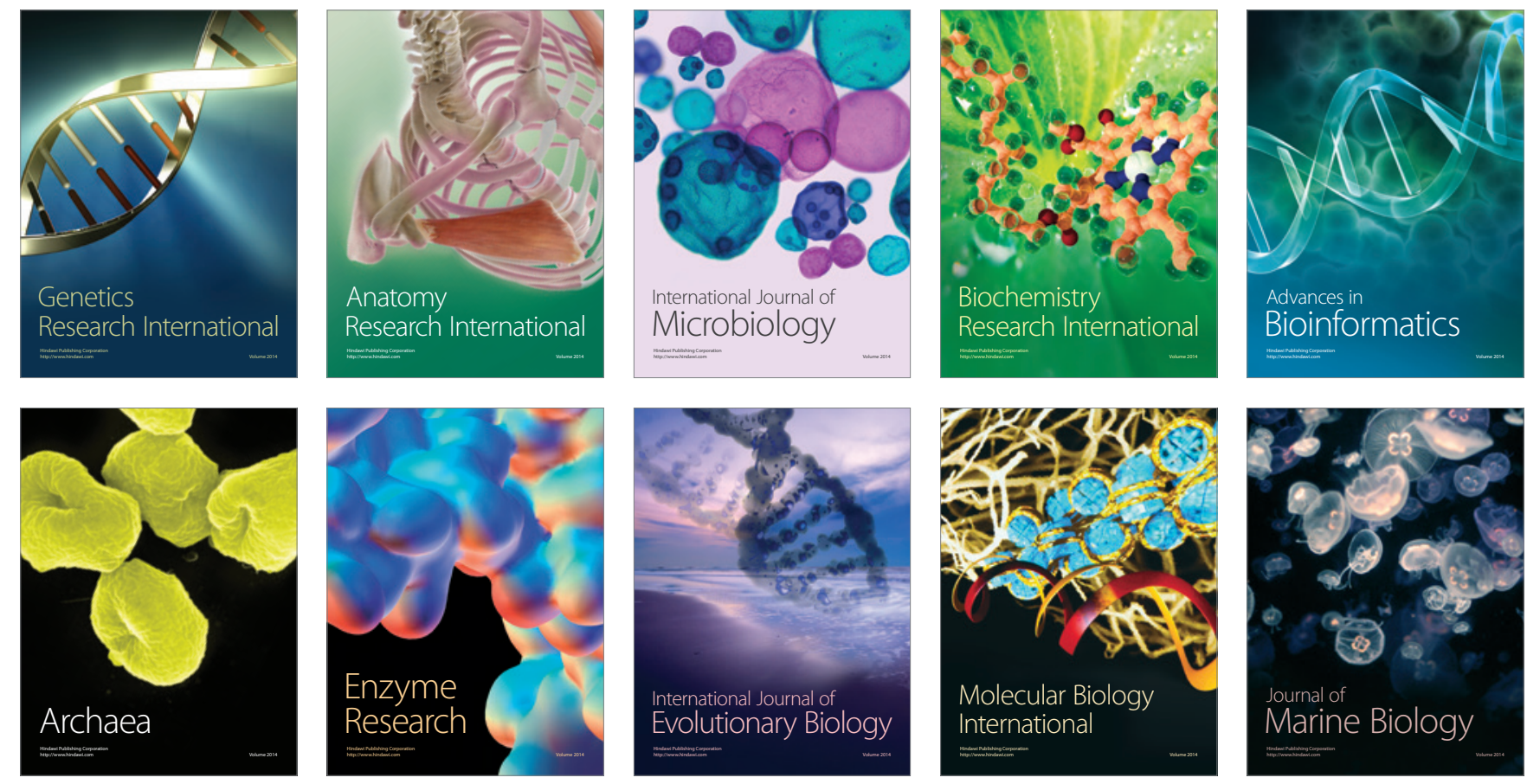